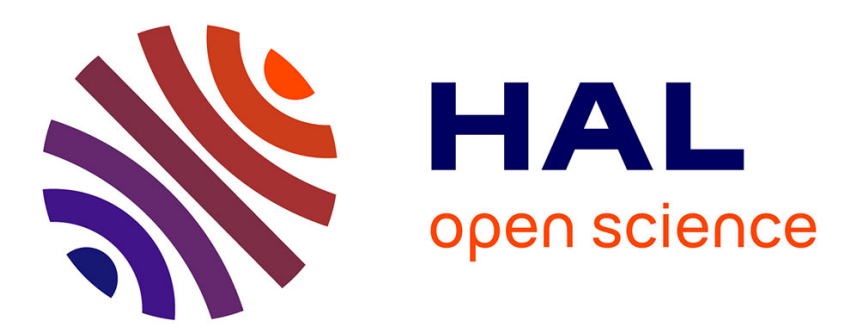

\title{
Well-Posedness of the Shooting Algorithm for State Constrained Optimal Control Problems with a Single Constraint and Control
}

\author{
J. Frederic Bonnans, Audrey Hermant
}

\section{- To cite this version:}

J. Frederic Bonnans, Audrey Hermant. Well-Posedness of the Shooting Algorithm for State Constrained Optimal Control Problems with a Single Constraint and Control. [Research Report] RR-5889, INRIA. 2006. inria-00071379

\section{HAL Id: inria-00071379 \\ https://hal.inria.fr/inria-00071379}

Submitted on 23 May 2006

HAL is a multi-disciplinary open access archive for the deposit and dissemination of scientific research documents, whether they are published or not. The documents may come from teaching and research institutions in France or abroad, or from public or private research centers.
L'archive ouverte pluridisciplinaire HAL, est destinée au dépôt et à la diffusion de documents scientifiques de niveau recherche, publiés ou non, émanant des établissements d'enseignement et de recherche français ou étrangers, des laboratoires publics ou privés. 
INSTITUT NATIONAL DE RECHERCHE EN INFORMATIQUE ET EN AUTOMATIQUE

\section{Well-Posedness of the Shooting Algorithm for State Constrained Optimal Control Problems with a Single Constraint and Control}

Frédéric Bonnans — Audrey Hermant

$\mathbf{N}^{\circ} \mathbf{5 8 8 9}$

April 2006

Thème NUM

apport

de recherche 



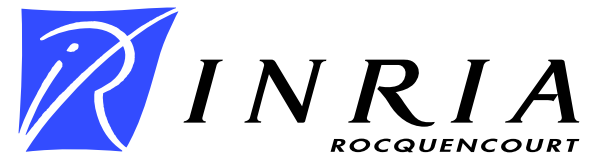

\title{
Well-Posedness of the Shooting Algorithm for State Constrained Optimal Control Problems with a Single Constraint and Control
}

\author{
Frédéric Bonnan团团, Audrey Hermant团† \\ Thème NUM — Systèmes numériques \\ Projet SYDOCO \\ Rapport de recherche $\mathrm{n}^{\circ} 5889$ - April 2006 - 36 pages
}

\begin{abstract}
This paper deals with the shooting algorithm for optimal control problems with a scalar control and a regular scalar state constraint. Additional conditions are displayed, under which the so-called alternative formulation is equivalent to Pontryagin's minimum principle. The shooting algorithm appears to be well-posed (invertible Jacobian), if and only if (i) the no-gap second order sufficient optimality condition holds, and (ii) when the constraint is of order $q \geq 3$, there is no boundary arc. Stability and sensitivity results without strict complementarity at touch points are derived using Robinson's strong regularity theory, under a minimal second-order sufficient condition. The directional derivatives of the control and state are obtained as solutions of a linear quadratic problem.
\end{abstract}

Key-words: Optimal control, Pontryagin's principle, state constraints, junction conditions, shooting algorithm, no-gap second-order optimality conditions, strong regularity, sensitivity analysis, directional derivatives.

* Frederic.Bonnans@inria.fr

$\dagger$ INRIA, B.P. 105, 78153 Le Chesnay, France

‡ Audrey.Hermant@inria.fr 


\section{Caractère bien posé de l'algorithme de tir pour les problèmes de commande optimale avec un contrôle scalaire et une contrainte sur l'état}

Résumé : Dans cet article, on étudie l'algorithme de tir pour les problèmes de commande optimale avec contraintes sur l'état. On donne les conditions supplémentaires nécessaires, sous lesquelles la formulation alternative est équivalente au Principe de Pontryaguine. On montre que l'algorithme de tir est bien posé, si et seulement si (i) une condition suffisante minimale du second ordre est satisfaite, et (ii) lorsque la contrainte est d'ordre $q \geq 3$, il n'y a pas d'arc frontière. Enfin, une analyse de stabilité et de sensibilité est effectuée, sans hypothèse de complémentarité stricte aux points de contacts isolés. On utilise pour ceci la théorie de la forte régularité de Robinson, dont on donne une caractérisation par une condition suffisante du second-ordre. Les dérivées directionnelles sont obtenues comme solution d'un problème linéaire quadratique.

Mots-clés : Commande optimale, Principe de Pontryaguine, contrainte sur l'état, conditions de jonctions, algorithme de tir, conditions d'optimalité du second ordre, régularité forte, analyse de sensibilité, dérivée directionnelle. 


\section{Introduction}

For optimal control problems satisfying the strengthened Legendre-Clebsch condition, Pontryagin's principle allows to express the control as a function of state and costate. For unconstrained problems, the resulting two points boundary value problem reduces to a finite dimensional "shooting" equation whose unknown is the initial costate (see e.g. [21]). The extension to control constrained problems is relatively easy, assuming nontangentiality conditions when a constraint becomes active or inactive. This approach allows to compute accurate solutions at low cost, once the structure of active constraints is known. For state constrained optimal control problems, a reformulation of the optimality conditions is needed, and the shooting equations take only into account some of the optimality conditions. Therefore, checking that the shooting equations are well-posed under minimal hypotheses becomes challenging.

An alternative formulation suitable for the shooting algorithm in presence of state constraints was first introduced by Bryson, Denham and Dreyfus [6, 5], in an heuristical manner. Some additional conditions (necessary for optimality) were missing, as was shown in Jackobson, Leyle and Speyer [13], where the first results on the regularity of the multiplier and on junctions conditions are stated. A significant clarification of their work can be found in Maurer in the unpublished paper [17], where the link between the results of [13] and the alternative formulation of [6, 5] is established. Numerous different versions of Pontryagin's Principle with state constraint were given in the literature; see [11. Stability results for first order state constraints were obtained by Dontchev and Hager [9, using an abstract Implicit Mapping Theorem in metric spaces. See also Malanowski [14, still for first-order constraints. Maurer and Malanowski obtain sensitivity results in [15] (first-order) and [16] (higher order), when strict complementarity holds, by application of the Implicit Function Theorem to the shooting mapping, and obtain derivatives as the solution of an equality constrained linear quadratic problem, but when the order of the constraint is $q \geq 2$, the data of the latter depend on the (precomputed) variation of entry times. Numerical applications of the shooting algorithm to state constrained problems in the aerospatial field are presented e.g. in [7, 2] and [18, where the role of additional conditions appears crucial to eliminate nonoptimal solutions; numerical examples of sensitivity analysis are given in [1].

This paper handles the case of a scalar control and a regular scalar state constraint, for which regularity and junctions conditions results are known. We assume that the Hamiltonian is uniformly strongly convex w.r.t. to the control variable, there are finitely many non tangential junctions times, and strict complementarity on boundary arcs holds.

We express the additional conditions under which the alternative formulation is equivalent to Pontryagin's principle. We prove that the shooting algorithm is well-posed (invertible Jacobian) iff (i) the no-gap second-order sufficient condition in [3] holds, and (ii) when the constraint is of order $q \geq 3$, there is no boundary arc. Then stability and sensitivity results, removing the strict complementarity hypothesis at touch points, are derived, applying Robinson's strong regularity theory [20] to the shooting mapping. We give a necessary and sufficient second-order condition characterizing the strong regularity property. The

$\mathrm{RR} \mathrm{n}^{\circ} 5889$ 
F. Bonnans \& A. Hermant

directional derivatives of the control and state are obtained as solutions of an inequality constrained linear quadratic problem, independent on the variations of junctions times.

The paper is organized as follow. In section 2] we give the characterization of Pontryagin extremals as solutions of the shooting equations under some minimal additional conditions. Then, in section 3] we give the characterization of the well-posedness of the shooting algorithm and the relation with the no-gap second-order optimality conditions obtained in [3]. Finally, in section 4 we give stability and sensitivity analysis results.

\section{Junctions Conditions}

Denote by $L^{\infty}(0, T)$ the Banach space of essentially bounded functions and by $W^{1, \infty}(0, T)$ the Sobolev space of functions having a weak derivative in $L^{\infty}(0, T)$. Let the control and state space be respectively $\mathcal{U}:=L^{\infty}([0, T] ; \mathbb{R})$ and $\mathcal{Y}:=W^{1, \infty}\left([0, T] ; \mathbb{R}^{n}\right)$. We consider the following optimal control problem with a scalar state constraint and a scalar control:

$$
\min _{(u, y) \in \mathcal{U} \times \mathcal{Y}} \int_{0}^{T} \ell(u(t), y(t)) \mathrm{d} t+\phi(y(T))
$$

subject to

$$
\begin{array}{cc}
\dot{y}(t)= & f(u(t), y(t)) \quad \text { a.e. } t \in[0, T] \quad ; \quad y(0)=y_{0} \\
g(y(t)) \leq 0 \quad \forall t \in[0, T] . &
\end{array}
$$

The data of the problem are the distributed cost $\ell: \mathbb{R} \times \mathbb{R}^{n} \rightarrow \mathbb{R}$, final cost $\phi: \mathbb{R}^{n} \rightarrow \mathbb{R}$, dynamics $f: \mathbb{R} \times \mathbb{R}^{n} \rightarrow \mathbb{R}^{n}$, state constraint $g: \mathbb{R}^{n} \rightarrow \mathbb{R}$, final time $T>0$, and initial condition $y_{0} \in \mathbb{R}^{n}$.

We assume throughout the paper that the following holds:

(A0) The mappings $\ell, \phi, f$ and $g$ are $k$-times continuously differentiable $\left(C^{k}\right)$ with $k \geq$ 2 , have locally Lipschitz continuous second-order derivatives, and the dynamics $f$ is Lipschitz continuous.

(A1) The initial condition satisfies $g\left(y_{0}\right)<0$.

The space of row vectors is denoted by $\mathbb{R}^{n *}$. The space of continuous functions over $[0, T]$ is denoted by $C[0, T]$. The dual space of Radon measures, denoted by $\mathcal{M}[0, T]$, is identified with the space of functions of bounded variation $B V(0, T)$ vanishing at zero. The transposition operator in $\mathbb{R}^{n}$ is denoted by a star *. Fréchet derivatives of $f$, $\ell$, etc. w.r.t. arguments $u \in \mathbb{R}, y \in \mathbb{R}^{n}$, are denoted by a subscript, for instance $f_{u}(u, y)=D_{u} f(u, y)$, $f_{u u}(u, y)=D_{u u}^{2} f(u, y)$. Total derivation w.r.t. to time is denoted by a dot, for example $\frac{\mathrm{d}}{\mathrm{d} t} g(y(t))=g_{y}(y(t)) \dot{y}(t)$.

A trajectory is an element $(u, y)$ of $\mathcal{U} \times \mathcal{Y}$ satisfying the state equation (2). Define the classical (resp. generalized) Hamiltonian functions of $(\mathcal{P}), H: \mathbb{R} \times \mathbb{R}^{n} \times \mathbb{R}^{n *} \rightarrow \mathbb{R}$ (resp. $\left.\mathcal{H}: \mathbb{R} \times \mathbb{R} \times \mathbb{R}^{n} \times \mathbb{R}^{n *} \rightarrow \mathbb{R}\right)$ by:

$$
H(u, y, p):=\ell(u, y)+p f(u, y) ; \quad \mathcal{H}\left(p_{0}, u, y, p\right):=p_{0} \ell(u, y)+p f(u, y) .
$$

INRIA 
First order necessary optimality conditions for $(\mathcal{P})$ are given by the Pontryagin Minimum Principle.

Definition 2.1. A trajectory $(u, y)$ is a Pontryagin extremal, if there exists $p_{0} \in \mathbb{R}^{+}$, $p \in B V\left([0, T] ; \mathbb{R}^{n *}\right)$ and $\eta \in \mathcal{M}[0, T]$, with $\left(p_{0}, \mathrm{~d} \eta\right) \neq 0$, such that:

$$
\begin{aligned}
\dot{y}(t) & =\mathcal{H}_{p}\left(p_{0}, u(t), y(t), p(t)\right) \quad \text { a.e. } t \in[0, T] \quad ; \quad y(0)=y_{0} \\
-\mathrm{d} p(t) & =\mathcal{H}_{y}\left(p_{0}, u(t), y(t), p(t)\right) \mathrm{d} t+g_{y}(y(t)) \mathrm{d} \eta(t) \quad \forall t \in[0, T] \\
p(T) & =p_{0} \phi_{y}(y(T)) \\
u(t) & \in \operatorname{argmin}_{w \in \mathbb{R}} \mathcal{H}\left(p_{0}, w, y(t), p(t)\right) \quad \text { a.e. } t \in[0, T] \\
g(y(t)) & \leq 0, \forall t \in[0, T] ; \quad \mathrm{d} \eta \geq 0 ; \quad \int_{0}^{T} g(y(t)) \mathrm{d} \eta(t)=0 .
\end{aligned}
$$

By $\mathrm{d} \eta \geq 0$, we mean that $\int_{0}^{T} \varphi(t) \mathrm{d} \eta(t) \geq 0$ for all nonnegative continuous function $\varphi \in C[0, T]$, or equivalently that $\eta$ is non decreasing. The costate equation (5) has the following signification:

$$
p(t)=\int_{t}^{T} \mathcal{H}_{y}\left(p_{0}, u(s), y(s), p(s)\right) \mathrm{d} s+\int_{t}^{T} g_{y}(y(s)) \mathrm{d} \eta(s)+\phi_{y}(y(T)) .
$$

The next Theorem is well-known (see [8, 10] for non differentiable versions).

Theorem 2.1. A trajectory $(u, y)$ solution of $(\mathcal{P})$ is a Pontryagin extremal.

Definitions A boundary (resp. interior) arc is a maximal interval of positive measure $\mathcal{I} \subset[0, T]$ such that $g(y(t))=0$ (resp. $g(y(t))<0$ ) for all $t \in \mathcal{I}$. If $\left[\tau_{e n}, \tau_{e x}\right]$ is a boundary arc, $\tau_{e n}$ and $\tau_{e x}$ are called entry and exit point, respectively. Entry and exit points are said to be regular if they are endpoint of an interior arc. A touch point $\tau$ in $(0, T)$ is an isolated contact point (endpoint of two interior arcs). Entry, exit and touch points are called junctions points (or times). We say that the junctions are regular, when the junctions points are regular. In this paper, only the case of finitely many regular junctions is dealt with.

The first-order time derivative of the state constraint along a trajectory $(u, y)$, i.e., $g^{(1)}(u, y)=\frac{\mathrm{d}}{\mathrm{d} t} g(y(t))=g_{y}(y) f(u, y)$, is denoted by $g^{(1)}(y)$ if the function $\mathbb{R} \times \mathbb{R}^{n} \rightarrow \mathbb{R}$; $(u, y) \mapsto g_{y}(y) f(u, y)$ does not depend on $u$ (that is, the function $(u, y) \mapsto g_{u}^{(1)}(u, y)$ is identically zero). We may define similarly $g^{(2)}, \ldots, g^{(q)}$ if $g, f$ are $C^{q}$ and if $g_{u}^{(j)} \equiv 0$, for all $j=1, \ldots, q-1$, and we have $g^{(j)}(u, y)=g_{y}^{(j-1)}(y) f(u, y)$, for $j=1, \ldots, q$.

Let $q \geq 1$ be the smallest number of times derivations of the state constraint, so that a dependence w.r.t. $u$ appears. If $q$ is finite, we say that $q$ is the order of the state constraint (see e.g. [6]). A state constraint of order $q$ is said to be regular along the trajectory $(u, y)$, if the condition below holds:

$$
\exists \gamma>0, \quad\left|g_{u}^{(q)}(\hat{u}, y(t))\right|>\gamma, \quad \text { for all } t \in[0, T] \text { and } \hat{u} \in \mathbb{R} .
$$


Note that the set of generalized multipliers $\left(p_{0}, p, \eta\right)$ is a cone. When $p_{0}=0$, we say that the multiplier is singular; otherwise it is said regular. Dividing then $(p, \eta)$ by $p_{0}$, we obtain the qualified version of Pontryagin's principle, substituting to the generalized Hamiltonian the classical Hamiltonian. It is easily seen that a Pontryagin extremal satisfying (9) and (A1) has no singular multiplier, and that the multiplier $(p, \eta)$ in the qualified version of Pontryagin's principle $\left(p_{0}=1\right)$ is unique.

Being of bounded variation, $p$ has at most countably many discontinuity times and has everywhere on $[0, T]$ left and right limits, denoted by $p\left(t^{ \pm}\right)=\lim _{t^{\prime} \rightarrow t^{ \pm}} p\left(t^{\prime}\right)$. The jump at $\tau \in(0, T)$ is denoted by $[p(\tau)]=p\left(\tau^{+}\right)-p\left(\tau^{-}\right)$. Similar conclusions hold for $\eta$.

Assumptions Let $(u, y)$ be a Pontryagin extremal with costate $p$ and multiplier $\eta$, satisfying Def. 2.1 (with $p_{0}=1$ ). We say that $(u, y)$ is a regular Pontryagin extremal, if assumptions (A2)-(A4) below are satisfied.

(A2) The Hamiltonian is strongly convex w.r.t. the control variable, uniformly w.r.t. $t \in$ $[0, T]$ :

$$
\exists \alpha>0, \quad H_{u u}\left(\hat{u}, y(t), p\left(t^{ \pm}\right)\right) \geq \alpha \quad \text { for all } t \in[0, T] \text { and } \hat{u} \in \mathbb{R} .
$$

(A3) The data of the problem are $C^{2 q}$, i.e. $k \geq 2 q$ in (A0), the state constraint is of order $q$ and regular, i.e. (9) holds.

(A4) The trajectory $\left(u, y_{u}\right)$ has a finite set of junctions times, that will be denoted by $\mathcal{T}=: \mathcal{T}_{\text {en }} \cup \mathcal{T}_{\text {ex }} \cup \mathcal{T}_{\text {to }}$, with $\mathcal{T}_{\text {en }}, \mathcal{T}_{\text {ex }}$ and $\mathcal{T}_{\text {to }}$ the disjoint (and possibly empty) subsets of respectively regular entry, exit and touch points, and we suppose that $g(y(T))<0$.

Hypothesis (A4) implies that all entry and exit points are regular junction points. In the sequel, we denote by $\mathcal{I}_{b}$ the union of boundary arcs, i.e. $\mathcal{I}_{b}:=\cup_{i=1}^{N_{b}}\left[\tau_{e n}^{i}, \tau_{e x}^{i}\right]$ for $\mathcal{T}_{e n}:=$ $\left\{\tau_{e n}^{1}<\cdots<\tau_{e n}^{N_{b}}\right\}$ and $\mathcal{T}_{e x}:=\left\{\tau_{e x}^{1}<\cdots<\tau_{e x}^{N_{b}}\right\}$.

Remark 2.1. In all the paper, (A3) can be weakened, replacing (9) by:

$$
\exists \gamma, \varepsilon>0, \quad\left|g_{u}^{(q)}(\hat{u}, y(t))\right|>\gamma, \quad \text { for all } t, \operatorname{dist}\left(t, \mathcal{I}_{b} \cup \mathcal{T}_{t o}\right)<\varepsilon \text { and } \hat{u} \in \mathbb{R} .
$$

Notations Given a finite subset $\mathcal{S}$ of $(0, T)$, we denote by $P C_{\mathcal{S}}^{k}[0, T]$ the set of functions over $[0, T]$ that are of class $C^{k}$ outside $\mathcal{S}$ ( $P C$ stands for piecewise continuous), and have, as well as their first $k$ derivatives, a left and right limit over $\mathcal{S} \cup\{0, T\}$.

Let $\varphi$ be a real-valued function over $[0, T]$. Assuming w.l.o.g. the elements of $\mathcal{S}$ in increasing order, we may define $\varphi(\mathcal{S}):=(\varphi(\tau))_{\tau \in \mathcal{S}} \in \mathbb{R}^{\text {Card } \mathcal{S}}$. We adopt a similar convention for vectors: $\nu_{\mathcal{S}}:=\left(\nu_{\tau}\right)_{\tau \in \mathcal{S}} \in \mathbb{R}^{\text {Card } \mathcal{S}}$, and will also use the following notations:

$$
\nu_{\mathcal{S}}^{1: q}:=\left(\begin{array}{c}
\nu_{\mathcal{S}}^{1} \\
\vdots \\
\nu_{\mathcal{S}}^{q}
\end{array}\right) \in \mathbb{R}^{q \operatorname{Card} \mathcal{S}} ; g^{(0: q-1)}(y(\mathcal{S})):=\left(\begin{array}{c}
g(y(\mathcal{S})) \\
\vdots \\
g^{(q-1)}(y(\mathcal{S}))
\end{array}\right) \in \mathbb{R}^{q \operatorname{Card} \mathcal{S}} .
$$

INRIA 


\subsection{Alternative Formulation of Optimality Conditions}

Under assumption (A4) we have a finite number of arcs and we can show, with regularity assumptions (A2)-(A3), that the multiplier $\eta$ is differentiable on the interior of each arc [13, 17]. An analysis of the optimality system on interiors of arcs shows then that a regular Pontryagin extremal satisfies the conditions stated in Proposition 2.1 below. An analysis at junctions times leads afterwards to junctions conditions given in Proposition 2.2

Proposition 2.1. Let $(u, y)$ be a regular Pontryagin extremal, satisfying (A2)-(A4). Then we have $u \in P C_{\mathcal{T}}^{q}[0, T], y \in P C_{\mathcal{T}}^{q}\left([0, T] ; \mathbb{R}^{n}\right)$ and there exists $p \in P C_{\mathcal{T}}^{1}\left([0, T] ; \mathbb{R}^{n *}\right), \eta_{0} \in$ $P C_{\mathcal{T}}^{0}[0, T]$, and jump parameters $\nu_{\mathcal{T}}$, such that the following optimality system is satisfied:

$$
\begin{array}{rlrl}
\dot{y}(t) & =H_{p}(u(t), y(t), p(t))=f(u(t), y(t)) & \text { on }[0, T] \backslash \mathcal{T} ; \quad y(0)=y_{0} \\
-\dot{p}(t) & =H_{y}(u(t), y(t), p(t))+g_{y}(y(t)) \eta_{0}(t) \quad \text { on }[0, T] \backslash \mathcal{T} \\
p(T) & =\phi_{y}(y(T)) & & \\
0 & =H_{u}(u(t), y(t), p(t)) & \text { on }[0, T] \backslash \mathcal{T} & \\
g(y(t)) & =0 \quad \text { on } \mathcal{I}_{b} & ; \quad \eta_{0}(t)=0 \quad \text { on }[0, T] \backslash \mathcal{I}_{b} \\
g(y(t)) & <0 \quad \text { on }[0, T] \backslash\left(\mathcal{I}_{b} \cup \mathcal{T}_{t o}\right) & ; \quad \eta_{0}(t) \geq 0 \quad \text { on } \operatorname{int} \mathcal{I}_{b} \\
g(y(\tau)) & =0 \quad \forall \tau \in \mathcal{T}_{t o} & & \\
{[p(\tau)]} & =-\nu_{\tau} g_{y}(y(\tau)) ; \nu_{\tau} \geq 0 \quad \forall \tau \in \mathcal{T} . &
\end{array}
$$

We denote by int $\mathcal{I}_{b}$ the interior of $\mathcal{I}_{b}$. A touch point $\tau \in \mathcal{T}_{\text {to }}$ is said to be essential, if $\nu_{\tau}>0$ in (19). We denote by $\mathcal{T}_{\text {to }}^{\text {ess }}$ the set of essential touch points. Hypotheses (A2)(A4) also imply the continuity of the control variable and of some of its time derivatives at junction points. The next proposition is due to Jacobson et al. [13. Its proof was later clarified in Maurer [17.

Proposition 2.2. Let $(u, y)$ be a regular Pontryagin extremal, satisfying (A2)-(A4). Then: (i) For all entry or exit point $\tau \in \mathcal{T}_{\text {en }} \cup \mathcal{T}_{\text {ex }}$ : (a) If $q$ is odd, $u$ and its $q-1$ first derivatives are continuous at $\tau, \nu_{\tau}=0$ and $p$ is continuous at $\tau$; (b) If $q$ is even, $u$ and its $q-2$ first derivatives are continuous at $\tau$.

(ii) For all touch point $\tau \in \mathcal{T}_{\text {to }}$ : (a) $u$ and its $q-2$ first derivatives are continuous at $\tau$; (b) If $\tau$ is non essential (i.e. $\nu_{\tau}=0$ ), $u$ and its $q$ first derivatives and $p$ are continuous at $\tau$; (c) if $q=1$, then $\tau$ is a non essential touch point.

Remark 2.2. If $(u, y)$ satisfies (A2)-(A4) and (12)-(19), the multiplier $\eta \in \mathcal{M}[0, T]$ such that $(u, y)$ satisfies definition 2.1 is given by:

$$
\mathrm{d} \eta(t)=\sum_{\tau \in \mathcal{T}} \nu_{\tau} \delta_{\tau}+\eta_{0}(t) \mathrm{d} t
$$

where $\delta_{\tau}$ denotes the Dirac measure at time $\tau, \nu_{\tau}=[\eta(\tau)]$ is the nonnegative jump at $\tau \in \mathcal{T}$, and the density $\eta_{0} \in P C_{\mathcal{T}}^{0}[0, T]$ equals $\frac{\mathrm{d} \eta}{\mathrm{d} t}$ almost everywhere. 
We now present the alternative formulation that will be used in the shooting algorithm. First introduced heuristically in [6, it is based on the use of the mixed explicit constraint $g^{(q)}(u(t), y(t))=0$ on boundary arcs. Let the augmented Hamiltonian $\tilde{H}: \mathbb{R} \times \mathbb{R}^{n} \times \mathbb{R}^{n *} \times$ $\mathbb{R} \mapsto \mathbb{R}$ be defined by:

$$
\tilde{H}\left(u, y, p_{q}, \eta_{q}\right)=H\left(u, y, p_{q}\right)+\eta_{q} g^{(q)}(u, y),
$$

where $q$ denotes the order of the state constraint and $H$ is the classical Hamiltonian (4).

Definition 2.2. We say that a trajectory $(u, y)$ satisfying (A3)-(A4) is solution of the alternative formulation, if there exist $p_{q} \in P C_{\mathcal{T}}^{q}\left([0, T] ; \mathbb{R}^{n *}\right), \eta_{q} \in P C_{\mathcal{T}}^{q}[0, T]$, alternative jump parameters $\nu_{\mathcal{T}_{\text {en }}}^{j}, j=1, \ldots, q$ and $\nu_{\mathcal{T}_{\text {to }}}$ such that the following relations are satisfied (we omit dependence in time):

$$
\begin{aligned}
\dot{y} & =\tilde{H}_{p}\left(u, y, p_{q}, \eta_{q}\right)=f(u, y) \quad \text { on }[0, T] \quad ; \quad y(0)=y_{0} \\
-\dot{p}_{q} & =\tilde{H}_{y}\left(u, y, p_{q}, \eta_{q}\right)=H_{y}\left(u, y, p_{q}\right)+\eta_{q} g_{y}^{(q)}(u, y) \quad \text { on }[0, T] \backslash \mathcal{T} \\
p_{q}(T) & =\phi_{y}(y(T)) \\
0 & =\tilde{H}_{u}\left(u, y, p_{q}, \eta_{q}\right)=H_{u}\left(u, y, p_{q}\right)+\eta_{q} g_{u}^{(q)}(u, y) \quad \text { on }[0, T] \backslash \mathcal{T} \\
g^{(j)}(y(\tau)) & =0 \quad \text { for } j=0,1, \ldots, q-1 ; \quad \tau \in \mathcal{T}_{\text {en }} \\
g^{(q)}(u, y) & =0 \quad \text { on } \mathcal{I}_{b} \\
g(y(\tau)) & =0 \quad \text { for all } \tau \in \mathcal{T}_{\text {to }} \\
\eta_{q}(t) & =0 \quad \text { on }[0, T] \backslash \mathcal{I}_{b} \\
{\left[p_{q}(\tau)\right] } & =-\sum_{j=1}^{q} \nu_{\tau}^{j} g_{y}^{(j-1)}(y(\tau)) \quad \text { for all } \tau \in \mathcal{T}_{\text {en }} \\
{\left[p_{q}(\tau)\right] } & =0 \quad \text { for all } \tau \in \mathcal{T}_{\text {ex }} \\
{\left[p_{q}(\tau)\right] } & =-\nu_{\tau} g_{y}(y(\tau)) \quad \text { for all } \tau \in \mathcal{T}_{\text {to }} .
\end{aligned}
$$

In the heuristical formulation of [6], equations (22)-(28) are interpreted as necessary optimality conditions for the problem of minimizing (1) subject to (2) and (26)-(28) for a fixed set of junctions times $\mathcal{T}$. Alternative jump parameters $\nu_{\tau_{e n}}^{1: q}$ appearing in (30) are seen as multipliers associated with the $q$ interior point constraints in [26] at a regular entry time $\tau_{\text {en }}$.

The assumption equivalent to (A2) for the alternative formulation, is the following, see remark 2.3(ii):

$(\mathbf{A 2})_{q} \exists \alpha>0, \tilde{H}_{u u}\left(\hat{u}, y(t), p_{q}\left(t^{ \pm}\right), \eta_{q}\left(t^{ \pm}\right)\right) \geq \alpha$, for all $t \in[0, T]$ and $\hat{u} \in \mathbb{R}$.

\subsection{Additional Conditions}

Relations (22)-(30) due to [6] are necessary, but not sufficient conditions for Pontryagin extremals. This was underlined in [13] where some additional necessary conditions were

INRIA 
provided, that allowed to show that a trajectory (with a fourth order state constraint) was not a Pontryagin extremal. We state in Prop. 2.3 the characterization of Pontryagin extremals based on the alternative formulation. We need some preliminary lemmas.

Lemma 2.1. Let $(u, y)$ a trajectory, and $\left(p_{q}, \eta_{q}\right) \in P C_{\mathcal{T}}^{1}\left([0, T], \mathbb{R}^{n *}\right) \times P C_{\mathcal{T}}^{0}[0, T]$ satisfying $\left(A 2_{q}\right)-(A 4)$ and (22)-(23), (25), (27). Then $\left(u, y, p_{q}, \eta_{q}\right)$ belongs to the set $P C_{\mathcal{T}}^{q}[0, T] \times$ $P C_{\mathcal{T}}^{q}\left([0, T] ; \mathbb{R}^{n}\right) \times P C_{\mathcal{T}}^{q}\left([0, T], \mathbb{R}^{n *}\right) \times P C_{\mathcal{T}}^{q}[0, T]$.

Proof. This is a consequence of the Implicit Function Theorem applied locally to (25) on interior arcs, and to (25) and (27) on boundary arcs.

Lemma 2.2. If constraint regularity (A3) holds along a trajectory $(u, y)$, and if $u \in$ $P C_{\mathcal{T}}^{q}[0, T]$, then, for all $t \in[0, T]$, vectors $\left(g_{y}(y(t)), \cdots, g_{y}^{(q-1)}(y(t))\right)$ are linearly independent (and hence, $q \leq n$ ).

Proof. Since $u \in P C_{\mathcal{T}}^{q}[0, T]$, the mappings $\left(A_{l}\right)_{0 \leq l \leq q-1}:[0, T] \backslash \mathcal{T} \mapsto \mathbb{R}^{n}$ defined inductively by:

$$
\left\{\begin{array}{l}
A_{0}(t):=f_{u}(u(t), y(t)) \\
A_{l}(t):=f_{y}(u(t), y(t)) A_{l-1}(t)-\dot{A}_{l-1}(t) \quad l=1, \ldots, q-1,
\end{array}\right.
$$

are well-defined, and $A_{l} \in P C_{\mathcal{T}}^{q-l}\left([0, T] ; \mathbb{R}^{n}\right)$ for $l=0, \ldots, q-1$. It has been showed in [17] that the following relations holds, for all $t \in[0, T]$ :

$$
\left\{\begin{array}{l}
g_{y}^{(j)}(y(t)) A_{l}\left(t^{ \pm}\right)=0 \quad \text { for } l=0, \ldots, q-2 ; j=0, \ldots, q-2-l \\
g_{u}^{(q)}\left(u\left(t^{ \pm}\right), y(t)\right)=g_{y}^{(q-l-1)}(y(t)) A_{l}\left(t^{ \pm}\right) \quad \text { for } l=0, \ldots, q-1
\end{array}\right.
$$

Set $C:=\left(g_{y}(y(t))^{*}, \cdots, g_{y}^{(q-1)}(y(t))^{*}\right)$. The above relations imply that the $q \times q$ matrix $D:=C^{\top}\left(A_{q-1}\left(t^{ \pm}\right), \ldots, A_{0}\left(t^{ \pm}\right)\right)$is lower triangular with nonzero diagonal elements equal to $g_{u}^{(q)}\left(u\left(t^{ \pm}\right), y(t)\right)$, hence has rank $q$. Therefore $C$ has rank at least $q$. The conclusion follows.

Proposition 2.3. Let $(u, y)$ a trajectory satisfying $\left(A 2_{q}\right)-(A 4)$ and the alternative formulation (22)-(28). Define the functions $\eta_{j}, 0 \leq j \leq q-1$, costate $p$ and jump parameters $\nu_{\mathcal{T}_{\text {en }}}$ and $\nu_{\mathcal{T}_{e x}}$ by:

$$
\begin{aligned}
\eta_{j}(t) & =(-1)^{q-j} \frac{\mathrm{d}^{q-j}}{\mathrm{~d} t^{q-j}} \eta_{q}(t) \quad \text { for } j=0, \ldots, q-1 ; t \in[0, T] \backslash \mathcal{T}, \\
p(t) & =p_{q}(t)+\sum_{j=1}^{q} \eta_{j}(t) g_{y}^{(j-1)}(y(t)) \quad t \in[0, T] \backslash \mathcal{T}, \\
\nu_{\tau_{e n}} & =\nu_{\tau_{e n}}^{1}-\eta_{1}\left(\tau_{e n}^{+}\right), \quad \forall \tau_{e n} \in \mathcal{T}_{e n} \quad ; \quad \nu_{\tau_{e x}}=\eta_{1}\left(\tau_{e x}^{-}\right), \quad \forall \tau_{e x} \in \mathcal{T}_{e x} .
\end{aligned}
$$

Then $(u, y)$ is a Pontryagin extremal that satisfies (12)-(19) iff all the following additional conditions are satisfied:

$$
\begin{aligned}
g(y(t)) & <0 & & \text { on }[0, T] \backslash\left(\mathcal{I}_{b} \cup \mathcal{I}_{\text {to }}\right) \\
\eta_{0}(t)=(-1)^{q} \frac{\mathrm{d}^{q}}{\mathrm{~d} t^{q}} \eta_{q}(t) & \geq 0 & & \text { on } \mathcal{I}_{b}
\end{aligned}
$$


At all entry time $\tau_{e n}$ :

$$
\left\{\begin{array}{l}
\nu_{\tau_{e n}}^{1}=\eta_{1}\left(\tau_{e n}^{+}\right) \quad \text { if } q \text { is odd } \\
\nu_{\tau_{e n}}^{1} \geq \eta_{1}\left(\tau_{e n}^{+}\right) \quad \text { if } q \text { is even }
\end{array} \quad ; \quad \nu_{\tau_{e n}}^{j}=\eta_{j}\left(\tau_{e n}^{+}\right) ; j=2, \ldots, q\right.
$$

At all exit time $\tau_{e x}$ :

$$
\left\{\begin{array}{l}
\eta_{1}\left(\tau_{e x}^{-}\right)=0 \quad \text { if } q \text { is odd } \\
\eta_{1}\left(\tau_{e x}^{-}\right) \geq 0 \quad \text { if } q \text { is even }
\end{array} \quad ; \quad \eta_{j}\left(\tau_{\text {ex }}^{-}\right)=0 ; j=2, \ldots, q .\right.
$$

At all touch time $\tau_{t o}$ :

$$
\nu_{\tau_{t o}} \geq 0
$$

Remark 2.3. (i) If $(u, y)$ is a regular Pontryagin extremal solution of (12)-(19), the functions $\eta_{j}, 1 \leq j \leq q$, costate $p_{q}$ and jump parameters $\nu_{\mathcal{T}_{e n}}^{1: q}$ such that $(u, y)$ satisfies the alternative formulation (22)-(28) and additional conditions (38)-(41) can be recovered from $p, \eta_{0}$ and $\nu_{\mathcal{T}}$ as follows. The functions $\eta_{j}$ are first given from (35) by successive integrations of $\eta_{0}$ over boundary arcs, with constant at exit times $\tau_{e x}$ given by (37) for $j=1$ and (41) for $j=2, \ldots, q$. Costate $p_{q}$ follows then from (36), and jump parameters at entry times $\nu_{T_{e n}}^{j}$ are given by (37) for $j=1$ and (40) for $j=2, \ldots, q$. Jumps parameters $\nu_{\mathcal{T}_{\text {to }}}$ associated with touch points are the same in both formulations.

(ii) Assumptions $(A 2)$ and $\left(A 2_{q}\right)$ are equivalent, since the constraint being of order $q$, we have:

$$
\begin{aligned}
\tilde{H}_{u u}\left(u, y, p_{q}, \eta_{q}\right) & =H_{u u}(u, y, p)-\sum_{j=1}^{q} \eta_{j}(t) g_{y}^{(j-1)}(y) f_{u u}(u, y)+\eta_{q} g_{u}^{(q)}(u, y) \\
& =H_{u u}(u, y, p)-\sum_{j=1}^{q-1} \eta_{j}(t) g_{u u}^{(j)}(y)(u, y)=H_{u u}(u, y, p) .
\end{aligned}
$$

Proof of Proposition 2.3. Since $\eta_{q}$ is piecewise $C^{q}$ by Lemma 2.1, functions $\eta_{j}, 0 \leq j \leq q-1$ are well-defined. We show the equivalence between equations (12)-(19) and equations (22)(32) augmented with (38)-(42).

Equivalence between state equations (12) and (22), final costate conditions (14) and (24), state constraint equations (16) and (26), (27), (29) on boundary arcs, (18) and (28) at touch points, is obvious. Equivalence between costate equations (13) and (23), and between control equations (15) and (25) follows from calculation, using the relations between the functions $\eta_{j}, p$ and $p_{q}$ and the fact that the state constraint is of order $q$ (see e.g. [17]).

Additional conditions are necessary to ensure equivalence between complementarity and junctions conditions. Obviously, (38)-(39) is equivalent to (17), as well as (32) and (42) to (19) for touch points. It remains to check that (19) is also equivalent to (30) and (31) at entry/exit points. Let $\tau_{e n} \in \mathcal{T}_{\text {en }}$. Expressing $\left[p_{q}\left(\tau_{e n}\right)\right]$ using on the one hand, the relationship (36) between $p$ and $p_{q}$, and on the other hand, jump condition (30), we obtain:

$$
\begin{aligned}
& {\left[p_{q}\left(\tau_{e n}\right)\right]=-\nu_{\tau_{e n}} g_{y}\left(y\left(\tau_{e n}\right)\right)-\sum_{j=1}^{q} \eta_{j}\left(\tau_{e n}^{+}\right) g_{y}^{(j-1)}\left(y\left(\tau_{e n}\right)\right),} \\
& {\left[p_{q}\left(\tau_{e n}\right)\right]=-\sum_{j=1}^{q} \nu_{\tau_{e n}}^{j} g_{y}^{(j-1)}\left(y\left(\tau_{e n}\right)\right) .}
\end{aligned}
$$


By Lemma 2.2] at $t=\tau_{e n}$, the right-hand sides of (43) and (44) are equal, iff the coefficients of $g_{y}^{(j-1)}\left(y\left(\tau_{e n}\right)\right)$ for $j=1, \ldots, q$ are equal. Eliminating $\nu_{\tau_{e n}}$ that must be non negative (and equals zero for odd order state constraints by Prop. 2.2), we deduce (40). Proceeding similarly at exit points, (41) follows.

Remark 2.4. Proposition [2.3 slightly improves section 5 of [17, in the sense that we give the complete set of additional conditions for which equivalence between Pontryagin's extremals and the alternative formulation holds.

Remark 2.5. Sign condition of $\eta_{q}^{(q)}$ on the boundary arc (39) and exit-point conditions (41) implies that the following necessary condition:

$$
(-1)^{q-j} \frac{\mathrm{d}^{q-j}}{\mathrm{~d} t^{q-j}} \eta_{q}(t)=\eta_{j}(t) \geq 0 \text { on } \mathcal{I}_{b} \quad \text { for } j=1, \ldots, q
$$

holds, as a consequence of (39) and (41). It is easily seen by induction, since $\dot{\eta}_{j}=-\eta_{j-1} \leq 0$ on $\mathcal{I}_{b}$ and $\eta_{j}\left(\tau_{e x}^{-}\right) \geq 0$, for all $\tau_{e x} \in \mathcal{T}_{e x}$. By [40, we deduce also that $\nu_{\tau_{e n}}^{j} \geq 0$, for all $\tau \in \mathcal{T}_{\text {en }}$ and $j=1, \ldots, q$.

\subsection{The shooting algorithm}

The shooting algorithm extracts from the necessary optimality conditions a finite dimensional set of equations (the shooting equations). If its Jacobian is invertible, we obtain a locally convergent algorithm by solving the shooting equations using, say, Newton's method.

In the unconstrained case, the initial value of the costate $p_{0}$ is mapped with the final condition (24). To handle alternative formulation of Def. 2.2. jump parameters and junctions times are introduced as shooting parameters. A given set of shooting parameters determines a unique trajectory $(u, y)$ and multipliers $\left(p_{q}, \eta_{q}\right)$ solution of the coupled state-costate system (22) - (23) with initial condition $p_{q}(0)=p_{0}$, algebraic equations (25)-(27), that give $u$ and $\eta_{q}$ as implicit functions of $\left(y, p_{q}\right)$ by (A2)-(A3), and jump conditions (30)-(32).

We use the shooting formulation of Maurer and Malanowski [15, 16]. Jump parameters $\nu_{\tau_{e n}}^{1: q}$ at an entry time $\tau_{e n}$, are associated with the $q$ interior points conditions (26). Necessary optimality conditions for entry and exit points $\tau_{e n}$ and $\tau_{e x}$ and touch points $\tau_{t o}$ (when $q \geq 2$ ) are as follows:

$$
\begin{aligned}
g^{(q)}\left(u\left(\tau_{e n}^{-}\right), y\left(\tau_{e n}\right)\right) & =0 \\
g^{(1)}\left(y\left(\tau_{\text {to }}\right)\right) & =0 .
\end{aligned} \quad ; \quad g^{(q)}\left(u\left(\tau_{\text {ex }}^{+}\right), y\left(\tau_{\text {ex }}\right)\right)=0
$$

By Proposition 2.2, the control is continuous along a regular Pontryagin extremal, so that (46) is a necessary optimality condition for entry/exit times. For a first order state constraint, we assume in the sequel that $\mathcal{T}_{t o}=\emptyset$ (see remark 2.9 below). Otherwise, since a touch point $\tau_{t o}$ is a local maximum of $g(y)$, (47) is a necessary optimality condition, and together with the interior point constraint (28), this gives two conditions associated with $\tau_{t o}$ and its jump parameter $\nu_{\tau_{t o}}$, for each $\tau_{t o} \in \mathcal{T}_{t o}$. 
Definition 2.3. A trajectory $(u, y)$ is a shooting extremal if it satisfies the alternative formulation (Def. [2.2) and conditions (46)- (47).

Let us show how (46) relates to the additional conditions of Prop. 2.3

Proposition 2.4. Let $(u, y)$ be a trajectory solution of the alternative formulation (22)-(28) and satisfying $\left(A 2_{q}\right)-(A 4)$. Then the two following conditions are equivalent:

(i) The control $u$ is continuous at entry/exit times $\tau_{e n}, \tau_{e x}$ (i.e., (46) holds);

(ii) The following subset of (40)- 41) holds:

$$
\eta_{q}\left(\tau_{e n}^{+}\right)-\nu_{\tau_{e n}}^{q}=0 \quad ; \quad \eta_{q}\left(\tau_{e x}^{-}\right)=0 .
$$

Proof. Let $\tau_{e n} \in \mathcal{T}_{\text {en }}$. By (A3), the function $\hat{u} \mapsto g^{(q)}\left(\hat{u}, y\left(\tau_{e n}\right)\right)$ is one-to-one. Since $g^{(q)}\left(u\left(\tau_{e n}^{+}\right), y\left(\tau_{e n}\right)\right)=0$, we have that $g^{(q)}\left(u\left(\tau_{e n}^{-}\right), y\left(\tau_{e n}\right)\right)=0$ iff the control is continuous at time $\tau_{e n}$; the same type of arguments holds for exit points. It follows that (46) is equivalent to the continuity of the control at entry/exit points.

By (25), we have

$$
\tilde{H}_{u}\left(u\left(\tau_{e n}^{-}\right), y\left(\tau_{e n}\right), p_{q}\left(\tau_{e n}^{-}\right), 0\right)=0=\tilde{H}_{u}\left(u\left(\tau_{e n}^{+}\right), y\left(\tau_{e n}\right), p_{q}\left(\tau_{e n}^{+}\right), \eta_{q}\left(\tau_{e n}^{+}\right)\right) .
$$

We abbreviate $u\left(\tau_{e n}^{-}\right)$to $u^{-}$and so on. Using the jump condition of the costate (30), it follows that:

$$
\tilde{H}_{u}\left(u^{+}, y, p_{q}^{+}, \eta_{q}^{+}\right)=H\left(u^{+}, y, p_{q}^{-}\right)-\sum_{j=1}^{q} \nu_{j} g_{y}^{(j-1)}(y) f_{u}\left(u^{+}, y\right)+\eta_{q}^{+} g^{(q)}\left(u^{+}, y\right) .
$$

The state constraint being of order $q$, we have $g_{y}^{(j-1)}(y) f_{u}(u, y)=g_{u}^{(j)}(y)=0$ for $j=$ $1, \ldots, q-1$, and hence, we obtain

$$
0=H_{u}\left(u^{+}, y, p_{q}^{-}\right)+\left(\eta_{q}^{+}-\nu_{q}\right) g_{u}^{(q)}\left(u^{+}, y\right) .
$$

Since $g_{u}^{(q)}\left(u^{+}, y\right) \neq 0$ by (A3), it follows that $H_{u}\left(u^{+}, y, p_{q}^{-}\right)=0$ iff $\eta_{q}^{+}=\nu_{q}$. Since by $\left(\mathrm{A} 2_{q}\right)$, $H_{u}\left(u^{+}, y, p_{q}^{-}\right)=0$ iff $u^{+}=u^{-}$, we deduce that $u$ is continuous at time $\tau_{e n}$ iff $\eta_{q}^{+}=\nu_{q}$. Similar arguments hold for exit points. The conclusion follows.

Remark 2.6. Since by (A2), $u$ is continuous a.e. on $[0, T]$ and has left-and right limits at all $t \in[0, T]$, it is sufficient in Proposition 2.4 to assume that (9) or (11) holds, for $\hat{u}$ in the segment $\left[u\left(t^{-}\right), u\left(t^{+}\right)\right]$(instead of $\mathbb{R}$ ), for all $t \in[0, T]$.

Remark 2.7. We can also check that if $(u, y)$ is a shooting extremal satisfying $\left(\mathrm{A} 2_{q}\right)-(\mathrm{A} 4)$, then $u$ is continuous at touch points $\tau \in \mathcal{T}_{t o}$, if $q \geq 2$. Indeed, (25), (29) and (32) lead to

$$
\begin{aligned}
0 & =H_{u}\left(u^{+}, y, p_{q}^{+}\right)-H_{u}\left(u^{-}, y, p_{q}^{-}\right) \\
& =H_{u}\left(u^{+}, y, p_{q}^{-}\right)-H_{u}\left(u^{-}, y, p_{q}^{-}\right)-\nu_{\tau} g_{y}(y) f_{u}\left(y, u^{+}\right) .
\end{aligned}
$$

Since $g_{y} f_{u}=g_{u}^{(1)} \equiv 0$ and $H_{u}\left(\cdot, y, p_{q}^{-}\right)$is one-to-one by $\left(\mathrm{A} 2_{q}\right)$, we obtain $u^{+}=u^{-}$. 
It follows that if $(u, y)$ is a shooting extremal satisfying $\left(\mathrm{A} 2_{q}\right)-(\mathrm{A} 4)$, then $u$ is continuous on $[0, T]$, still assuming that $\mathcal{T}_{t o}=\emptyset$ if $q=1$ (see remark [2.9).

The structure of a trajectory is defined as the (finite) number of boundary arcs and touch points of the trajectory, and the order in which they occur w.r.t. time. Assuming the structure of the optimal trajectory to be known, the shooting mapping is defined as follows. Denote by $N_{b}$ and $N_{t o}$ the number of boundary arcs and touch points of the trajectory, respectively. The space of shooting parameters is:

$$
\Theta:=\mathbb{R}^{n} \times \mathbb{R}^{q N_{b}} \times \mathbb{R}^{N_{t o}} \times \mathbb{R}^{N_{b}} \times \mathbb{R}^{N_{b}} \times \mathbb{R}^{N_{t_{o}}} .
$$

With the above notations, and for a given order of boundary arcs and touch points, the shooting mapping $\mathcal{F}$ is defined over a neighborhood in $\Theta$ of shooting parameters associated with a regular Pontryagin extremal, into $\Theta$, by:

$$
\theta=\left(\begin{array}{c}
p_{0}^{*} \\
\nu_{\mathcal{T}_{\text {en }}}^{1: q} \\
\nu_{\mathcal{T}_{\text {to }}} \\
\mathcal{T}_{\text {en }} \\
\mathcal{T}_{\text {ex }} \\
\mathcal{T}_{\text {to }}
\end{array}\right) \mapsto\left(\begin{array}{c}
p_{q}(T)^{*}-\phi_{y}(y(T))^{*} \\
g^{(0: q-1)}\left(y\left(\mathcal{T}_{\text {en }}\right)\right) \\
g\left(y\left(\mathcal{T}_{\text {to }}\right)\right) \\
g^{(q)}\left(u\left(\mathcal{T}_{\text {en }}^{-}\right), y\left(\mathcal{T}_{\text {en }}\right)\right) \\
g^{(q)}\left(u\left(\mathcal{T}_{\text {ex }}^{+}\right), y\left(\mathcal{T}_{\text {ex }}\right)\right) \\
g^{(1)}\left(y\left(\mathcal{T}_{\text {to }}\right)\right)
\end{array}\right)
$$

By construction, a zero of the shooting mapping $\mathcal{F}$ provides a trajectory $(u, y)$ that is a shooting extremal. In view of Propositions 2.3 and 2.4 the following holds:

Corollary 2.1. A shooting extremal is a Pontryagin extremal iff it satisfies the following minimal additional conditions: (38) on interior arcs, (39) on boundary arcs, (42) at touch points, and for all entry point $\tau_{\text {en }} \in \mathcal{T}_{\text {en }}$ and exit point $\tau_{e x} \in \mathcal{T}_{\text {ex }}$ :

$$
\begin{aligned}
& \text { if } q \geq 2 \text { is even: } \nu_{\tau_{e n}}^{1}-(-1)^{q-1} \eta_{q}^{(q-1)}\left(\tau_{e n}^{+}\right) \geq 0 ;(-1)^{q-1} \eta_{q}^{(q-1)}\left(\tau_{e x}^{-}\right) \geq 0 ;(50) \\
& \left\{\begin{array}{c}
\text { if } q \geq 3 \text { is odd, } j=1, \ldots, q-1, \text { and if } q \geq 4 \text { is even, } j=2, \ldots, q-1: \\
\nu_{\tau_{e n}}^{j}-(-1)^{q-j} \eta_{q}^{(-j)}\left(\tau_{e n}^{+}\right)=0 \quad ; \quad(-1)^{q-j} \eta_{q}^{(q-j)}\left(\tau_{e x}^{-}\right)=0 .
\end{array}\right.
\end{aligned}
$$

Remark 2.8. It follows that for first and second-order state constraints, and for constraints of order $q>2$ having no boundary arcs (see remark 4.2 concerning existence of boundary arcs for state constraints of order $q \geq 3$ ), the additional conditions reduce to the inequalities (38), (39), (42), and also (50) when $q=2$ at entry/exit points.

Remark 2.9. For a first-order state constraint, jump parameters $\nu_{\mathcal{T}_{t o}}$ associated with touch points are equal to zero along a Pontryagin extremal by Prop. 2.2 So we may ignore touch points in the shooting algorithm, and for this reason, we assume in this paper that $\mathcal{T}_{\text {to }}=\emptyset$ if $q=1$. 


\section{Well-Posedness of the Shooting Algorithm}

We say that the shooting algorithm is well-posed, if the Jacobian of the shooting mapping (49) is invertible at some local solution of $(\mathcal{P})$. This allows to apply locally a Newton method in order to find a shooting extremal; the additional conditions for a Pontryagin extremal have to be checked afterwards.

Let us first give some definitions. Given $u \in \mathcal{U}$, denote by $y_{u}$ the (unique) solution of state equation (2). This well-defined mapping is of class $C^{k}$ under assumption (A0). Let the cost function be:

$$
J(u)=\int_{0}^{T} \ell\left(u(t), y_{u}(t)\right) \mathrm{d} t+\phi\left(y_{u}(T)\right) .
$$

We say that a trajectory $\left(u, y=y_{u}\right)$ is a local optimal solution of $(\mathcal{P})$, satisfying the quadratic growth condition, if there exists $c, r>0$ such that:

$$
J(\tilde{u}) \geq J(u)+c\|\tilde{u}-u\|_{2}^{2} \quad \forall \tilde{u} \in B_{\infty}(u, r) ; g(y \tilde{u}(t)) \leq 0 \text { on }[0, T],
$$

where $B_{\infty}$ denotes the open ball in $L^{\infty}(0, T)$ with center $u$ and radius $r$. This condition involves two norms, $L^{\infty}(0, T)$ for the neighborhood, and $L^{2}(0, T)$ for the growth condition.

Let $(u, y)$ be a regular Pontryagin extremal. We make the strict complementarity assumption (compare to (39), (42) and (50):

(A5) (i) For all boundary $\operatorname{arc}\left[\tau_{e n}, \tau_{e x}\right]$ :

$$
(-1)^{q} \frac{\mathrm{d}^{q}}{\mathrm{~d} t^{q}} \eta_{q}(t)>0 \quad \text { a.e. on }\left(\tau_{e n}, \tau_{e x}\right)
$$

$$
\begin{array}{lcl}
\text { If } q \text { is odd: } & \frac{\mathrm{d}^{q}}{\mathrm{~d} t^{q}} \eta_{q}\left(\tau_{e n}^{+}\right)<0 & ; \frac{\mathrm{d}^{q}}{\mathrm{~d} t^{q}} \eta_{q}\left(\tau_{e x}^{-}\right)<0, \\
\text { If } q \text { is even }: & \nu_{\tau_{e n}}^{1}+\frac{\mathrm{d}^{q-1}}{\mathrm{~d} t^{q-1}} \eta_{q}\left(\tau_{e n}^{+}\right)>0 & ; \frac{\mathrm{d}^{q-1}}{\mathrm{~d} t^{q-1}} \eta_{q}\left(\tau_{e x}^{-}\right)<0 .
\end{array}
$$

(ii) For all touch point $\tau_{t o} \in \mathcal{T}_{\text {to }}$ :

$$
\nu_{\tau_{t o}}>0
$$

Recall that $(-1)^{q} \frac{\mathrm{d}^{q}}{\mathrm{~d} t^{q}} \eta_{q}(t)$ equals $\eta_{0}$, the density of $\eta$ (see Prop. 2.3). Let $\hat{q}:=2 q-1$ if $q$ is odd and $\hat{q}:=2 q-2$ if $q$ is even. By Prop. 2.2, $\hat{q}+1$ is the smallest possible order for which the corresponding derivative of $g(y)$ may be nonzero at a jonction point. Note that $\hat{q}=q$ for $q=1,2$.

Lemma 3.1. Let $(u, y)$ be a regular Pontryagin extremal satisfying (A2)-(A4). Assumptions (55) and (56) holds respectively when $q$ is odd and even, iff the following non tangentiality condition at order $\hat{q}+1$ holds: for all entry time $\tau_{\text {en }} \in \mathcal{T}_{\text {en }}$ and all exit time $\tau_{\text {ex }} \in \mathcal{T}_{\text {ex }}$,

$$
\left.(-1)^{\hat{q}+1} \frac{\mathrm{d}^{\hat{q}+1}}{\mathrm{~d} t^{\hat{q}+1}} g(y(t))\right|_{t=\tau_{e n}^{-}}<0 ;\left.\quad \frac{\mathrm{d}^{\hat{q}+1}}{\mathrm{~d} t^{\hat{q}+1}} g(y(t))\right|_{t=\tau_{e x}^{+}}<0 .
$$


Proof. By Prop. 2.3 (see (37)), (56) is equivalent, when $q$ is even, to say that $\nu_{\tau}>0$ at entry/exit points $\tau \in \mathcal{T}_{\text {en }} \cup \mathcal{T}_{\text {ex }}$. The conclusion is then a consequence of Prop. 2.3 and Lemma A.2 whose (technical) proof is given in the Appendix.

Assumption (A5)(ii) implies, if $q=1$, that $\mathcal{T}_{\text {to }}=\emptyset$ by Prop. 2.2(ii). When $q \geq 2$, we assume that all touch points of $(u, y)$ are reducible, in the following sense:

(A6) For all touch point $\tau_{t o} \in \mathcal{T}_{t o}$ :

$$
\left.\frac{\mathrm{d}^{2}}{\mathrm{~d} t^{2}} g(y(t))\right|_{t=\tau_{t o}}<0 .
$$

This makes sense, since when $q \geq 2$, we have $\frac{\mathrm{d}^{2}}{\mathrm{~d}^{2}} g(y(t))=g^{(2)}(u, y)$ and $u$ is continuous by Prop. 2.2

\subsection{Statement of main results}

Define the quadratic cost function:

$$
\begin{gathered}
\mathcal{J}_{q}(v, z):=\int_{0}^{T} \tilde{H}_{(u, y),(u, y)}\left(u, y, p_{q}, \eta_{q}\right)((v, z),(v, z)) \mathrm{d} t \\
+z(T)^{*} \phi_{y y}(y(T)) z(T)+\sum_{\tau \in \mathcal{T}_{e n}} \sum_{j=1}^{q} \nu_{\tau}^{j} z(\tau)^{*} g_{y y}^{(j-1)}(y(\tau)) z(\tau) \\
+\sum_{\tau \in \mathcal{T}_{t o}} \nu_{\tau}\left(z(\tau)^{*} g_{y y}(y(\tau)) z(\tau)-\frac{\left(g_{y}^{(1)}(y(\tau)) z(\tau)\right)^{2}}{\left.\frac{\mathrm{d}}{\mathrm{d} t} g^{(1)}(y(t))\right|_{t=\tau}}\right)
\end{gathered}
$$

where $\tilde{H}$ is the augmented Hamiltonian [21, and the set of constraints:

$$
\begin{array}{rl}
\dot{z}=f_{y}(u, y) z+f_{u}(u, y) v & \text { on }[0, T] \quad ; \quad z(0)=0 \\
g_{y}^{(j)}(y(\tau)) z(\tau)=0 & \text { for } j=0, \ldots, q-1 ; \tau \in \mathcal{T}_{\text {en }} \\
g_{(u, y)}^{(q)}(u(t), y(t))(v(t), z(t))=0 & t \in \mathcal{I}_{b} \\
g_{y}(y(\tau)) z(\tau)=0 & \tau \in \mathcal{T}_{\text {to }} .
\end{array}
$$

Since the state equation and constraints are linear, and the cost function is quadratic, all with bounded coefficients, we may take as linearized control and state spaces $\mathcal{V}:=L^{2}(0, T)$ and $\mathcal{Z}:=H^{1}\left(0, T ; \mathbb{R}^{n}\right)$, where $H^{1}(0, T)$ is the Sobolev space of functions in $L^{2}(0, T)$ with weak derivatives in $L^{2}(0, T)$. Let the Linear Quadratic Problem $\left(P Q_{q}\right)$ be defined by:

$$
\left(\mathbf{P Q}_{q}\right) \quad \min _{(v, z) \in \mathcal{V} \times \mathcal{Z}} \frac{1}{2} \mathcal{J}_{q}(v, z) \quad \text { subject to (61)- (64). }
$$

Consider the following second-order conditions:

$$
\begin{aligned}
& (v, z)=0 \text { is solution of }\left(P Q_{q}\right) . \\
& (v, z)=0 \text { is the unique solution of }\left(P Q_{q}\right) .
\end{aligned}
$$


F. Bonnans \& A. Hermant

Theorem 3.1 (No-gap second order optimality conditions). (i) Let (u,y) be a local solution of $(\mathcal{P})$ satisfying (A2)-(A6). Then its associated multipliers in the alternative formulation are such that the second-order necessary condition (66) holds.

(ii) Let $(u, y)$ be a Pontryagin extremal satisfying (A2)-(A6). Then the second-order suffcient condition (67) holds iff $(u, y)$ is a local optimal solution of $(\mathcal{P})$ satisfying the quadratic growth condition (53).

Theorem 3.2 (Well-Poseness of the Shooting Algorithm). Let $(u, y)$ be a local optimal solution of $(\mathcal{P})$ satisfying (A2)-(A6). Then the shooting algorithm is well-posed, iff the two conditions below hold: (i) If $q \geq 3$, the trajectory $(u, y)$ does not have boundary arcs; (ii) The second-order sufficient condition (67) holds.

In general, even for unconstrained problems, the invertibility of the Jacobian of the shooting mapping at a Pontryagin extremal does not imply that the second-order sufficient condition (67) holds. We comment the ill-posedness of the shooting algorithm along boundary arc of order $q \geq 3$ in remark 4.2 .

Combining Th. 3.1(ii) and 3.2, we obtain that if $(u, y)$ is a local optimal solution of $(\mathcal{P})$ satisfying (A2)-(A6) and condition (i) of Th. 3.2, then the shooting algorithm is well-posed iff $(u, y)$ satisfy the quadratic growth condition.

\subsection{Proof of the no-gap Second-order Optimality Conditions (The- orem 3.1)}

We use the no-gap second order optimality conditions established in 3 . Let $(u, y)$ be a regular Pontryagin extremal, with the multiplier $\eta \in \mathcal{M}[0, T]$ given by (20). Consider the quadratic cost function:

$$
\begin{aligned}
\mathcal{J}(v, z):= & \int_{0}^{T} H_{(u, y),(u, y)}(u, y, p)((v, z),(v, z)) \mathrm{d} t+z(T)^{*} \phi_{y y}(y(T)) z(T) \\
& +\int_{0}^{T}\left(z^{*} g_{y y}(y) z\right) \mathrm{d} \eta-\sum_{\tau \in \mathcal{T}_{t o}} \nu_{\tau} \frac{\left(g_{y}^{(1)}(y(\tau)) z(\tau)\right)^{2}}{\left.\frac{\mathrm{d}}{\mathrm{d} t} g^{(1)}(y(t))\right|_{t=\tau}}
\end{aligned}
$$

where $H$ is the classical Hamiltonian (4), and the constraint

$$
g_{y}(y(t)) z(t)=0 \quad \text { on } \mathcal{I}_{b} \cup \mathcal{T}_{\text {to }} .
$$

The quadratic problem used in the formulation of second-order optimality condition in $[3]$ is the following:

$$
\text { (PQ) } \min _{(v, z) \in \mathcal{V} \times \mathcal{Z}} \frac{1}{2} \mathcal{J}(v, z) \quad \text { subject to 61, 69. }
$$

Theorem 3.3. (i) If $(u, y)$ is an optimal solution of $(\mathcal{P})$ such that (A2)-(A6) hold, then $(v, z)=0$ is solution of problem (70). 
(ii) If $(u, y)$ is a Pontryagin extremal such that (A2)-(A6) hold, it is a local optimal solution of $(\mathcal{P})$ satisfying the quadratic growth condition (53) iff problem (70) has zero for unique solution.

Proof. See Corollary 3.2 and Theorems 4.1 and 5.1 in [3].

We establish the link between Th. 3.3 and the second-order conditions (66)- (67) derived from the alternative formulation. In the end of this section we often omit the time argument when there is no ambiguity. The proof of the next lemma is easy and omitted.

Lemma 3.2. Assume that the state constraint is of order $q$. Then for all trajectory $(u, y)$ and all linearized trajectory $(v, z) \in \mathcal{V} \times \mathcal{Z}$ satisfying (61), the following holds:

$$
\begin{aligned}
\frac{\mathrm{d}^{j}}{\mathrm{~d} t^{j}} g_{y}(y(t)) z(t) & =g_{y}^{(j)}(u, y) z, \quad j=1, \ldots, q-1, \\
\frac{\mathrm{d}^{q}}{\mathrm{~d} t^{q}} g_{y}(y(t)) z(t) & =g_{y}^{(q)}(u, y) z+g_{u}^{(q)}(u, y) v .
\end{aligned}
$$

Lemma 3.3. Let $(u, y)$ be a regular Pontryagin extremal, with classical and alternative multipliers $(p, \eta)$ and $\left(p_{q}, \eta_{q}, \nu_{\mathcal{T}_{\text {en }}}^{1: q}, \nu_{\mathcal{T}_{\text {to }}}\right)$, respectively, related to each other by (35)-(37), (40) and (20). Then the quadratic cost functions $\mathcal{J}$ and $\mathcal{J}_{q}$, defined respectively in (68) and (60), are equal to each other over the space of the linearized trajectories $(v, z) \in \mathcal{V} \times \mathcal{Z}$ satisfying (61).

Proof. Let $(v, z) \in \mathcal{V} \times \mathcal{Z}$ satisfy (61) and set $\Delta_{P Q}:=\mathcal{J}(v, z)-\mathcal{J}_{q}(v, z)$. Using (20), it is easily seen that the terms corresponding to the touch points and final time vanish, hence we get:

$$
\begin{aligned}
\Delta_{P Q}= & \int_{0}^{T}\left(p-p_{q}\right) D^{2} f(u, y)((v, z),(v, z)) \mathrm{d} t+\int_{0}^{T} g_{y y}(y)(z, z) \eta_{0}(t) \mathrm{d} t \\
& -\int_{0}^{T} D^{2} g^{(q)}(u, y)((v, z),(v, z)) \eta_{q}(t) \mathrm{d} t+\sum_{\tau \in \mathcal{T}_{\text {ex }}} \nu_{\tau} g_{y y}(y)(z, z)(\tau) \\
& +\sum_{\tau \in \mathcal{T}_{\text {en }}}\left(\nu_{\tau} g_{y y}(y)(z, z)(\tau)-\sum_{j=1}^{q} \nu_{\tau}^{j} g_{y y}^{(j-1)}(y)(z, z)(\tau)\right) .
\end{aligned}
$$

In the sequel we abbreviate the notation $((v, z),(v, z))$ by $((v, z))^{2}$. Relations (35)-(36) between $p$ and $p_{q}$ lead to

$$
\begin{aligned}
\Delta_{P Q}= & \sum_{j=1}^{q} \int_{0}^{T} g_{y}^{(j-1)}(y) D^{2} f(u, y)((v, z))^{2} \eta_{j}(t) \mathrm{d} t+\int_{0}^{T} g_{y y}(y)(z, z) \eta_{0}(t) \mathrm{d} t \\
& -\int_{0}^{T} D^{2} g^{(q)}(u, y)((v, z))^{2} \eta_{q}(t) \mathrm{d} t+\sum_{\tau \in \mathcal{T}_{\text {ex }}} \nu_{\tau} g_{y y}(y)(z, z)(\tau)
\end{aligned}
$$

$\mathrm{RR} \quad \mathrm{n}^{\circ} 5889$ 


$$
+\sum_{\tau \in \mathcal{T}_{\text {en }}}\left(\nu_{\tau} g_{y y}(y)(z, z)(\tau)-\sum_{j=1}^{q} \nu_{\tau}^{j} g_{y y}^{(j-1)}(y)(z, z)(\tau)\right) .
$$

The constraint being of order $q$, we have $g^{(j)}(u, y)=g_{y}^{(j-1)} f(u, y)$ for $j=0, \ldots, q-1$. It follows that

$$
\begin{aligned}
D^{2} g^{(j)}(u, y)((v, z))^{2}= & g_{y y y}^{(j-1)}(f(u, y), z, z)+2 g_{y y}^{(j-1)}(z, D f(u, y)(v, z)) \\
& +g_{y}^{(j-1)} D^{2} f(u, y)((v, z))^{2} .
\end{aligned}
$$

In addition, by the linearized state equation (61), we have, for all $j=1, \ldots, q$ :

$$
\frac{\mathrm{d}}{\mathrm{d} t} g_{y y}^{(j-1)}(y(t))(z(t), z(t))=g_{y y y}^{(j-1)}(y)(f(u, y), z, z)+2 g_{y y}^{(j-1)}(y)(z, D f(u, y)(v, z)),
$$

which gives by (174), for $j=1, \ldots, q$ :

$$
\frac{\mathrm{d}}{\mathrm{d} t} g_{y y}^{(j-1)}(y(t))(z(t), z(t))=D^{2} g^{(j)}(u, y)((v, z))^{2}-g_{y}^{(j-1)}(y) D^{2} f(u, y)((v, z))^{2} .
$$

Since $g_{u}^{(j-1)}(u, y) \equiv 0$ for $j=1, \ldots, q$, we have $g_{y y}^{(j-1)}(y)(z, z)=D^{2} g^{(j-1)}(u, y)((v, z))^{2}$ for $j=1, \ldots, q$. Multiplying (75) by $\eta_{j}$, integrating over $[0, T]$ and integrating by parts the left-hand side (recall that $\dot{\eta}_{j}=-\eta_{j-1}$ ), we obtain, for $j=1, \ldots, q$ :

$$
\begin{gathered}
\int_{0}^{T} D^{2} g^{(j-1)}(u, y)((v, z))^{2} \eta_{j-1}(t) \mathrm{d} t+\sum_{\tau \in \tau_{e x}} g_{y y}^{(j-1)}(y)(z, z) \eta_{j}\left(\tau^{-}\right) \\
-\sum_{\tau \in \tau_{e n}} g_{y y}^{(j-1)}(y)(z, z) \eta_{j}\left(\tau^{+}\right)= \\
\int_{0}^{T} D^{2} g^{(j)}(u, y)((v, z))^{2} \eta_{j}(t)-\int_{0}^{T} g_{y}^{(j-1)} D^{2} f(u, y)((v, z))^{2} \eta_{j}(t) \mathrm{d} t .
\end{gathered}
$$

Adding the above equalities for $j=1, \ldots, q$, we get after simplification by the terms $\int_{0}^{T} D^{2} g^{(j)}(u, y)((v, z))^{2} \eta_{j}$ for $j=1, \ldots, q-1$ that:

$$
\begin{gathered}
\int_{0}^{T} g_{y y}(y)(z, z) \eta_{0}(t) \mathrm{d} t+\sum_{j=1}^{q} \sum_{\tau \in \tau_{e x}} g_{y y}^{(j-1)}(y)(z, z) \eta_{j}\left(\tau^{-}\right) \\
-\sum_{j=1}^{q} \sum_{\tau \in \tau_{e n}} g_{y y}^{(j-1)}(y)(z, z) \eta_{j}\left(\tau^{+}\right)= \\
\int_{0}^{T} D^{2} g^{(q)}(u, y)((v, z))^{2} \eta_{q}(t)-\sum_{j=1}^{q} \int_{0}^{T} g_{y}^{(j-1)} D^{2} f(u, y)((v, z))^{2} \eta_{j}(t) \mathrm{d} t .
\end{gathered}
$$

INRIA 
Substituting into (73) gives:

$$
\begin{aligned}
\Delta_{P Q} & =\sum_{\tau \in \tau_{e x}}\left(\nu_{\tau} g_{y y}(y)(z, z)(\tau)-\sum_{j=1}^{q} g_{y y}^{(j-1)}(y)(z, z) \eta_{j}\left(\tau^{-}\right)\right) \\
& +\sum_{\tau \in \tau_{e n}}\left(\nu_{\tau} g_{y y}(y)(z, z)(\tau)+\sum_{j=1}^{q}\left(\eta_{j}\left(\tau^{+}\right)-\nu_{\tau}^{j}\right) g_{y y}^{(j-1)}(y)(z, z)(\tau)\right) .
\end{aligned}
$$

Using (37) and additional conditions at entry and exit points (40)-(41), we obtain that $\Delta_{P Q}=0$. Thus, the cost functions of the two quadratic problems coincide on the feasible set.

Proof of Theorem 3.1. The state constraint being of order $q$, it follows from (71)-(72) that (62) - (64) and (69) are equivalent. By Lemma 3.3, problems $\left(P Q_{q}\right)$ and (70) have the same feasible set and cost function on that feasible set, and hence, they also have the same value and same set of optimal solutions. The conclusion follows then from Theorem 3.3

\subsection{Proof of the Well-posedness (Theorem [3.2)}

We give a sequence of lemmas; some of them will also be used in section 4 . We denote by $g_{y}^{(j)}\left(y\left(\mathcal{T}_{\text {en }}\right)\right) z\left(\mathcal{T}_{\text {en }}\right), g_{(u, y)}^{(q)}\left(u\left(\mathcal{T}_{\text {ex }}\right), y\left(\mathcal{T}_{\text {ex }}\right)\right)\left(v\left(\mathcal{T}_{\text {ex }}^{+}\right), z\left(\mathcal{T}_{\text {ex }}\right)\right)$, the vectors in $\mathbb{R}^{N_{b}}$ of components $g_{y}^{(j)}(y(\tau)) z(\tau), g_{(u, y)}^{(q)}(u(\tau), y(\tau))\left(v\left(\tau^{+}\right), z(\tau)\right)$ respectively, for $\tau \in \mathcal{T}_{\text {en }}$. We denote by $g_{y}^{(0: q-1)}\left(y\left(\mathcal{T}_{\text {en }}\right)\right) z\left(\mathcal{T}_{\text {en }}\right)$ the vector in $\mathbb{R}^{q N_{b}}$ of component $g_{y}^{(j)}\left(y\left(\mathcal{T}_{\text {en }}\right)\right) z\left(\mathcal{T}_{\text {en }}\right), 0 \leq j \leq q-1$, $\tau \in \mathcal{T}_{\text {en }}$.

Lemma 3.4. Let $\theta_{0}=\left(p_{0}^{*}, \nu_{\mathcal{T}_{\text {en }}}^{1: q}, \nu_{\mathcal{T}_{\text {to }}}, \mathcal{T}_{\text {en }}, \mathcal{T}_{\text {ex }}, \mathcal{T}_{\text {to }}\right) \in \Theta$ be such that $\mathcal{F}\left(\theta_{0}\right)=0$ with the shooting mapping $\mathcal{F}$ defined in [49). Then $\mathcal{F}$ is of class $C^{1}$ on a neighborhood $\Theta_{0}$ of $\theta_{0}$, and at the direction

$$
\omega:=\left(\pi_{0}^{*}, \gamma_{\mathcal{T}_{\text {en }}}^{1: q}, \gamma_{\mathcal{T}_{\text {to }}}, \sigma_{\mathcal{T}_{\text {en }}}, \sigma_{\mathcal{T}_{\text {ex }}}, \sigma_{\mathcal{T}_{\text {to }}}\right) \in \Theta
$$

the vector $\mathcal{M}:=D \mathcal{F}\left(\theta_{0}\right) \omega$ splits into $\mathcal{M}=\left(\mathcal{M}_{\mathcal{Q}}^{*}, \mathcal{M}_{\mathcal{T}}^{*}\right)^{*}$ given by:

$$
\begin{gathered}
\mathcal{M}_{\mathcal{Q}}:=\left(\begin{array}{c}
\pi(T)^{*}-\phi_{y y}(y(T)) z(T) \\
g_{y}^{(0: q-1)}\left(y\left(\mathcal{T}_{\text {en }}\right)\right) z\left(\mathcal{T}_{\text {en }}\right) \\
g_{y}\left(y\left(\mathcal{T}_{\text {to }}\right)\right) z\left(\mathcal{T}_{\text {to }}\right)
\end{array}\right) \\
\mathcal{M}_{\mathcal{T}}:=\left(\begin{array}{c}
g_{(u, y)}^{(q)}\left(u\left(\mathcal{T}_{\text {en }}\right), y\left(\mathcal{T}_{\text {en }}\right)\right)\left(v\left(\mathcal{T}_{\text {en }}^{-}\right), z\left(\mathcal{T}_{\text {en }}\right)\right)+\left.\sigma_{\mathcal{T}_{\text {en }}} \frac{\mathrm{d}}{\mathrm{d} t} g^{(q)}(u, y)\right|_{t=\mathcal{T}_{\text {en }}^{-}} \\
g_{(u, y)}^{(q)}\left(u\left(\mathcal{T}_{\text {ex }}\right), y\left(\mathcal{T}_{\text {ex }}\right)\right)\left(v\left(\mathcal{T}_{\text {ex }}^{+}\right), z\left(\mathcal{T}_{\text {ex }}\right)\right)+\left.\sigma_{\mathcal{T}_{\text {ex }}} \frac{\mathrm{d}}{\mathrm{d} t} g^{(q)}(u, y)\right|_{t=\mathcal{T}_{\text {ex }}^{+}} \\
g_{y}^{(1)}\left(y\left(\mathcal{T}_{\text {to }}\right)\right) z\left(\mathcal{T}_{t o}\right)+\left.\sigma_{\mathcal{T}_{t o}} \frac{\mathrm{d}}{\mathrm{d} t} g^{(1)}(y)\right|_{t=\mathcal{T}_{t o}}
\end{array}\right),
\end{gathered}
$$


where $(v, z, \pi, \zeta)$, the linearized control, state, costate and state constraint multiplier, are solutions of (omitting arguments $\left(u, y, p_{q}, \eta_{q}\right)$ and $t$ )

$$
\begin{aligned}
\dot{z} & =f_{y} z+f_{u} v & & \text { on }[0, T] ; \\
-\dot{\pi} & =\tilde{H}_{y y} z+\tilde{H}_{y u} v+\pi f_{y}+\zeta g_{y}^{(q)} & & \text { on }[0, T] \backslash \mathcal{T} \\
0 & =\tilde{H}_{u y} z+\tilde{H}_{u u} v+\pi f_{u}+\zeta g_{u}^{(q)} & & \text { a.e. on }[0, T] \\
0 & =g_{y}^{(q)} z+g_{u}^{(q)} v & & \text { a.e. on } \mathcal{I}_{b} \\
0 & =\zeta & & \text { on }[0, T] \backslash \mathcal{I}_{b}
\end{aligned}
$$

with initial and junctions conditions of $\pi$ given by $\pi(0)=\pi_{0}$, and

$$
\begin{aligned}
& {[\pi(\tau)]=-\sum_{j=1}^{q} \nu_{\tau}^{j} z(\tau)^{*} g_{y y}^{(j-1)}(y(\tau))-\sum_{j=1}^{q} \gamma_{\tau}^{j} g_{y}^{(j-1)}(y(\tau))} \\
& \quad-\sigma_{\tau} \sum_{j=1}^{q-1} \nu_{\tau}^{j} g_{y}^{(j)}(y(\tau)) \quad ; \quad \tau \in \mathcal{T}_{\text {en }} \\
& {[\pi(\tau)]=0 \quad ; \quad \tau \in \mathcal{T}_{\text {ex }}} \\
& {[\pi(\tau)]=-\nu_{\tau} z(\tau)^{*} g_{y y}(y(\tau))-\gamma_{\tau} g_{y}(y(\tau))-\sigma_{\tau} \nu_{\tau} g_{y}^{(1)}(y(\tau)) \quad ; \quad \tau \in \mathcal{T}_{\text {to }} .}
\end{aligned}
$$

Proof. We detail only how we obtain the jumps conditions of the linearized costate $\pi$ at entry times, the others equations being obvious. In view of [30, it is easy to check that the jump of $\pi$ at $\tau \in \mathcal{T}_{\text {en }}$ is given by:

$$
[\pi(\tau)]=-\sum_{j=1}^{q} \nu_{\tau}^{j} z(\tau)^{*} g_{y y}(y(\tau))-\sum_{j=1}^{q} \gamma_{\tau}^{j} g_{y}(y(\tau))-\sigma_{\tau} \Delta_{\tau},
$$

where the vector of sensitivity coefficients $\Delta_{\tau}$ on junction time is given by:

$$
\Delta_{\tau}=-\sum_{j=1}^{q} \nu_{\tau}^{j} g_{y y}^{(j-1)}(y(\tau)) f\left(u\left(\tau^{-}\right), y(\tau)\right)-\left[\tilde{H}_{y}\left(u(\tau), y(\tau), p_{q}(\tau), \eta_{q}(\tau)\right)\right] .
$$

By continuity of $u$ at junctions times and (30), we have (omitting argument $\tau$ and setting $\left.\eta_{q}^{+}=\eta_{q}\left(\tau^{+}\right)\right)$:

$$
\Delta_{\tau}=-\sum_{j=1}^{q} \nu_{\tau}^{j} g_{y y}^{(j-1)}(y) f(u, y)-\sum_{j=1}^{q} \nu_{\tau}^{j} g_{y}^{(j-1)}(y) f_{y}(u, y)+\eta_{q}^{+} g_{y}^{(q)}(u, y) .
$$

Since $g_{y}^{(j)}(u, y)=g_{y y}^{(j-1)}(y) f(u, y)+g_{y}^{(j-1)}(y) f_{y}(u, y)$ for $j=1, \ldots, q$, and by Prop. 2.4 $\eta_{q}\left(\tau^{+}\right)=\nu_{\tau}^{q}$, we obtain 84 . 
We recall that a continuous quadratic form defined over a Hilbert space is a Legendre form (see e.g. [12, 4), if it is weakly lower semi-continuous, and satisfies the following property: for all weakly convergent sequence $\left(v_{n}\right) \subset L^{2}(0, T), v_{n} \rightarrow v$, we have $Q\left(v_{n}\right) \rightarrow Q(v)$ iff $v_{n} \rightarrow v$ strongly.

Lemma 3.5. Let $(u, y)$ be a shooting extremal, satisfying $\left(A 2_{q}\right)-(A 4)$. For all $v \in \mathcal{V}$, define $z_{v}$ as the (unique) solution in $\mathcal{Z}$ of the linearized state equation (61), and the operator $\mathcal{A}: \mathcal{V} \rightarrow W:=L^{2}\left(\mathcal{I}_{b}\right) \times \mathbb{R}^{q N_{b}} \times \mathbb{R}^{N_{t o}}$ by

$$
\mathcal{A} v=\left(\begin{array}{c}
g_{y}^{(q)}(u(\cdot), y(\cdot)) z_{v}(\cdot)+\left.g_{u}^{(q)}(u(\cdot), y(\cdot)) v(\cdot)\right|_{\mathcal{I}_{b}} \\
g_{y}^{(0: q-1)}\left(y\left(\mathcal{T}_{\text {en }}\right)\right) z_{v}\left(\mathcal{T}_{\text {en }}\right) \\
g_{y}\left(y\left(\mathcal{T}_{\text {to }}\right)\right) z_{v}\left(\mathcal{T}_{\text {to }}\right)
\end{array}\right) .
$$

Then: (i) The continuous linear operator $\mathcal{A}$ is onto. (ii) Assume that the second-order sufficient condition (67) holds. Then there exists $\alpha>0$, such that

$$
Q(v):=\mathcal{J}_{q}\left(v, z_{v}\right) \geq \alpha\|v\|_{2}^{2}, \quad \forall v \in \operatorname{Ker} \mathcal{A} .
$$

By $\left.\varphi\right|_{\mathcal{I}_{b}}$, we denote the restriction to $\mathcal{I}_{b}$ of function $\varphi$ defined over $[0, T]$.

Proof. The continuity of $\mathcal{A}$ follows from that of $\mathcal{V} \rightarrow \mathcal{Z}, v \mapsto z_{v}$. By (9) and Lemma 3.2 the range of the mapping $\mathcal{V} \rightarrow \mathcal{Z}, v \mapsto g_{y}(y(\cdot)) z_{v}(\cdot)$, is the subspace denoted by $H_{0}^{q}$ of functions $\varphi \in H^{q}(0, T)=W^{q, 2}(0, T)$ satisfying $\varphi^{(j)}(0)=0$ for all $j=0, \ldots, q-1$. Points (i) follows, since by (A4), for all $\left(\psi(\cdot), b_{\mathcal{T}_{\text {en }}}^{1:}, b_{\mathcal{T}_{\text {to }}}\right) \in W$, there exists $\varphi \in H_{0}^{q}$ such that $\varphi^{(q)}(t)=\psi(t)$ a.e. on $\mathcal{I}_{b}, \varphi^{(j-1)}\left(\mathcal{T}_{\text {en }}\right)=b_{\mathcal{T}_{\text {en }}}^{j}, j=1, \ldots, q$, and $\varphi\left(\mathcal{T}_{t o}\right)=b_{\mathcal{T}_{t o}}$.

By $\left(\mathrm{A} 2_{q}\right)$, we can show that $Q(v)$ is a Legendre form over $L^{2}(0, T)$ (the proof is similar to Lemma 4.3 in [3]). By (67), we have $Q(v)>0$ for all $v \in \operatorname{Ker} \mathcal{A}$, which implies (88) by Lemma A.3

Proposition 3.1. Let $(u, y)$ be a shooting extremal and denote by $\theta_{0} \in \Theta$ its set of shooting parameters. Assume that: (i) The second-order sufficient condition (67) is satisfied; (ii) The following holds at junctions times:

$$
\begin{gathered}
\left.\frac{\mathrm{d}}{\mathrm{d} t} g^{(q)}(u, y)\right|_{t=\tau^{-}} \neq 0 \quad \forall \tau \in \mathcal{T}_{\text {en }} ;\left.\frac{\mathrm{d}}{\mathrm{d} t} g^{(q)}(u, y)\right|_{t=\tau^{+}} \neq 0 \quad \forall \tau \in \mathcal{T}_{\text {ex }} \\
\left.\frac{\mathrm{d}}{\mathrm{d} t} g^{(1)}(y)\right|_{t=\tau_{t o}} \neq 0 \quad \forall \tau \in \mathcal{T}_{\text {to }} .
\end{gathered}
$$

Then the Jacobian $D \mathcal{F}\left(\theta_{0}\right)$ of the shooting mapping is invertible, and for all $\delta \in \Theta, \delta=$ $\left(a_{T}, b_{\mathcal{T}_{e n}}^{1: q}, b_{\mathcal{T}_{t o}}, c_{\mathcal{T}_{\text {en }}}, c_{\mathcal{T}_{\text {ex }}}, c_{\mathcal{T}_{\text {to }}}\right)$, the (unique) solution $\omega \in \Theta$ of $D \mathcal{F}\left(\theta_{0}\right) \omega=\delta$, with $\omega$ given by (76), is as follows. With the notations of Lemma [3.5. denote by $\left(u_{\delta}, w_{\delta}\right)$ with $w_{\delta}=$ $\left(\zeta_{\delta}, \lambda_{\delta, \mathcal{T}_{e n}}^{1: q}, \lambda_{\delta, \mathcal{T}_{t o}}\right)$ the unique solution in $L^{2}(0, T) \times W$ of the first-order optimality system of the problem:

$$
\begin{gathered}
\left(\mathcal{P}^{\delta}\right) \min _{v \in \mathcal{V}} \frac{1}{2} \mathcal{J}_{q}\left(v, z_{v}\right)+a_{T}^{*} z_{v}(T)+\sum_{\tau \in \mathcal{T}_{t o}} c_{\tau} \nu_{\tau} \frac{g_{y}^{(1)}(y(\tau)) z_{v}(\tau)}{\left.\frac{\mathrm{d}}{\mathrm{d} t} g^{(1)}(y)\right|_{t=\tau}}, \\
\text { subject to } \\
\mathcal{A} v=\left(0_{L_{2}\left(\mathcal{I}_{b}\right)}, b_{\mathcal{T}_{\text {en }}}^{1: q}, b_{\mathcal{T}_{\text {to }}}\right)^{*}
\end{gathered}
$$


Then: $\pi_{0}=\pi_{\delta}(0)$, where $\pi_{\delta}$ is solution on $[0, T] \backslash \mathcal{T}$ of (80) with $\left(v_{\delta}, \zeta_{\delta}, z_{v_{\delta}}\right)$, final and jumps conditions of $\pi_{\delta}$ being given by:

$$
\begin{aligned}
\pi_{\delta}(T) & =z_{\delta}(T)^{*} \phi_{y y}(y(T))+a_{T}^{*}, \\
-\left[\pi_{\delta}(\tau)\right] & =\sum_{j=1}^{q} \nu_{\tau}^{j} z_{\delta}(\tau)^{*} g_{y y}^{(j-1)}(y(\tau))+\sum_{j=1}^{q} \lambda_{\delta, \tau}^{j} g_{y}^{(j-1)}(y(\tau)), \quad \tau \in \mathcal{T}_{e n}, \\
-\left[\pi_{\delta}(\tau)\right] & =0, \quad \tau \in \mathcal{T}_{e x}, \\
-\left[\pi_{\delta}(\tau)\right] & =\nu_{\tau} z_{\delta}(\tau)^{*} g_{y y}(y(\tau))+\lambda_{\delta, \tau} g_{y}(y(\tau)) \\
& -\nu_{\tau} z_{\delta}(\tau)^{*} \frac{g_{y}^{(1)}(y(\tau))^{*} g_{y}^{(1)}(y(\tau))}{\left.\frac{\mathrm{d}}{\mathrm{d} t} g^{(1)}(y)\right|_{t=\tau}}+c_{\tau} \nu_{\tau} \frac{g_{y}^{(1)}(y(\tau))}{\left.\frac{\mathrm{d}}{\mathrm{d} t} g^{(1)}(y)\right|_{t=\tau}}, \quad \tau \in \mathcal{T}_{\text {to }} ;
\end{aligned}
$$

and we have: $\gamma_{\mathcal{T}_{\text {to }}}=\lambda_{\delta, \mathcal{T}_{\text {to }}}$,

$$
\begin{aligned}
\sigma_{\tau} & =\frac{c_{\tau}-g_{y}^{(1)}(y(\tau)) z_{\delta}(\tau)}{\left.\frac{\mathrm{d}}{\mathrm{d} t} g^{(1)}(y)\right|_{t=\tau}}, \quad \tau \in \mathcal{T}_{t o}, \\
\sigma_{\tau} & =\frac{c_{\tau}-g_{(u, y)}^{(q)}(u(\tau), y(\tau))\left(v_{\delta}\left(\tau^{+}\right), z_{\delta}(\tau)\right)}{\left.\frac{\mathrm{d}}{\mathrm{d} t} g^{(q)}(u, y)\right|_{t=\tau^{+}}}, \quad \tau \in \mathcal{T}_{e x}, \\
\sigma_{\tau} & =\frac{c_{\tau}-g_{(u, y)}^{(q)}(u(\tau), y(\tau))\left(v_{\delta}\left(\tau^{-}\right), z_{\delta}(\tau)\right)}{\left.\frac{\mathrm{d}}{\mathrm{d} t} g^{(q)}(u, y)\right|_{t=\tau^{-}}}, \quad \tau \in \mathcal{T}_{e n}, \\
\gamma_{\tau}^{1} & =\lambda_{\delta, \tau}^{1}, \quad \gamma_{\tau}^{j}=\lambda_{\delta, \tau}^{j}-\nu_{\tau}^{j-1} \sigma_{\tau}, \quad j=2, \ldots, q, \quad \tau \in \mathcal{T}_{\text {en }} .
\end{aligned}
$$

Note that $\left(v_{\delta}, \zeta_{\delta}, z_{v_{\delta}}, \pi_{\delta}\right)$ satisfies (79)-(83). It follows by $\left(\mathrm{A} 2_{q}\right)$ and (9) that $\left(v_{\delta}, \zeta_{\delta}\right) \in$ $P C_{\mathcal{T}}^{q}[0, T]$, and hence, $v_{\delta}$ has limits when $t \rightarrow \tau^{-}$and $t \rightarrow \tau^{+}$, for $\tau$ in respectively $\mathcal{T}_{\text {en }}$ and $\mathcal{T}_{\text {ex }}$, so (97)-98 make sense.

Remark 3.1. Note that (58) implies (89) when $q=1,2$, since $\hat{q}=q$.

Remark 3.2. The above proposition is an explicit elimination property, valid for any order $q \geq 1$, that enables to express the solution $\omega$ of $D \mathcal{F}\left(\theta_{0}\right) \omega=\delta$ in function of the optimal solution and multipliers of the quadratic problem $\left(\mathcal{P}^{\delta}\right)$, independent on the variations of junctions times. In the case $q=1$, the term in factor of variation of entry time $\sigma_{\tau}$ in 84 is zero, so that Lemma 3.1 is nothing but the block decoupling property of the Jacobian already established in [15]. In the case $q \geq 2$, our result differs from the one in [16], since the two authors use a quadratic problem depending on the variation of the entry point, leading to an additional assumption (A.11).

Proof. Assume that (i)-(ii) hold and let $\delta \in \Theta$. Let $\omega \in \Theta$ be solution of $D \mathcal{F}\left(\theta_{0}\right) \omega=\delta$. By Lemma 3.4, (90) and the last row of (78) enable to eliminate the variations $\sigma_{\mathcal{T}_{t o}}$ of touch points, so that we obtain (96). Then, by Lemma 3.4 again, substituting $\sigma_{\tau}$ into (86) gives (95), with $\lambda_{\delta, \mathcal{T}_{t o}}=\gamma_{\mathcal{T}_{t o}}$. We recognize with (77), (79)- 85] and (95) the first order optimality 
conditions of $\left(\mathcal{P}_{\delta}\right)$, where the multiplier associated with (64) is $\gamma_{\mathcal{T}_{t o}}$ and the multiplier $\lambda_{\delta, \mathcal{T}_{\text {en }}}^{j}$, $j=1, \ldots, q$, associated with entry points constraint (62) satisfy (99). Finally, by (89) and the two first rows of (78), we get (98)-(97). By Lemma 3.5. Lemma A.4 (with $r=0$ ) implies that the first-order optimality system of $\left(\mathcal{P}_{\delta}\right)$ has a unique solution and multipliers. Hence for all $\delta \in \Theta, \omega$ exists and is uniquely determined by the above relations. It follows that the Jacobian of the shooting mapping is onto, and hence invertible.

Proof of Theorem 3.2. Since (A5)(i) implies, by Lemma 3.1 that (58) holds, (89) is satisfied when $q=1,2$ (see Rem. [3.1), or trivially when the trajectory $(u, y)$ has no boundary arc, i.e. $\mathcal{T}_{\text {en }}=\mathcal{T}_{\text {ex }}=\emptyset$. With (A6) and the second-order sufficient condition [67, the invertibility of the Jacobian of the shooting mapping follows from Prop. 3.1.

Let us show now the converse. Assume first that (i) does not hold, i.e. $q \geq 3$ and $(u, y)$ has a boundary arc. By Prop. [2.2(i), $\dot{u}$ is continuous at junctions times $\tau_{e n}$ and $\tau_{e x}$. Therefore, the function $\frac{\mathrm{d}}{\mathrm{d} t} g^{(q)}(u(t), y(t))$ depending on $(y, u, \dot{u})$ is also continuous at entry and exit times and vanishes on the boundary arc, so that (89) does not hold, at none of the regular entry/exit times. Then it is easily seen by Lemma 3.4 that we can find some non zero $\tilde{\omega} \in \Theta$ such that $D \mathcal{F}\left(\theta_{0}\right) \tilde{\omega}=0$. Indeed, take e.g. $\tilde{\sigma}_{\tau} \neq 0$ for $\tau \in \mathcal{T}_{e x}$, and all other components of $\tilde{\omega}$ equal to zero. It follows that the Jacobian of the shooting mapping is singular.

Assume now that (i) is satisfied but (ii) is not. Since $(u, y)$ is an optimal solution of $(\mathcal{P})$, by Th. 3.1 the second-order necessary condition (66) is satisfied, hence the value of problem $\left(P Q_{q}\right)$ is zero and $(v, z)=0$ is solution. Since the second-order sufficient condition (67) does not hold, there exists another optimal solution $\left(\tilde{v}_{0}, \tilde{z}_{0}\right) \neq 0$ of $\left(P Q_{q}\right)$, and hence a non zero solution of its first-order optimality conditions (61)- (64), (79)- (83), with final and jump conditions of the associated costate $\tilde{\pi}_{0}$ given by (92)- (95) with $a_{T}=0$, and multipliers $\left(\tilde{\lambda}_{\mathcal{T}_{\text {en }}}^{1: q}, \tilde{\lambda}_{\mathcal{T}_{\text {to }}}\right)$ associated respectively with (62) and (64).

Setting $\tilde{\pi}_{0}:=\tilde{\pi}_{0}(0)$, we claim that $\left(\tilde{\pi}_{0}, \tilde{\lambda}_{\mathcal{T}_{\text {en }}}^{1: q}, \tilde{\lambda}_{\mathcal{T}_{\text {to }}}\right) \neq 0$. Indeed, suppose that all of them were zero. Eliminating $u$ by 81 as a linear function of $(z, \pi)$, and integrating from $(z(0), \pi(0))=0$ over the first arc the linear differential equations (179)-(80), we would have $(z, \pi, v, \zeta)=0$, until the first junction time. All the jump parameters $\tilde{\lambda}_{\mathcal{T}_{\text {en }}}^{j}$ and $\tilde{\lambda}_{\mathcal{T}_{\text {to }}}$ being equal to zero, eliminating on boundary $\operatorname{arcs} \zeta$ by (83), we would have $\left(\tilde{z}_{0}, \tilde{\pi}_{0}, \tilde{v}_{0}, \tilde{\zeta}_{0}\right)=0$ over $[0, T]$, which leads to a contradiction.

Let now $\tilde{\gamma}_{\mathcal{T}_{t o}}=\tilde{\lambda}_{\mathcal{T}_{t o}}$ and $\left(\tilde{\sigma}_{\mathcal{T}}, \tilde{\gamma}_{\mathcal{T}_{e n}}^{1: q}\right)$ be solution of (96)- with $c_{\mathcal{T}}=0$. We have $\tilde{\omega}:=\left(\tilde{\pi}_{0}, \tilde{\gamma}_{\mathcal{T}_{e n}}^{1: q}, \tilde{\gamma}_{\mathcal{T}_{t o}}, \tilde{\sigma}_{\mathcal{T}_{e n}}, \tilde{\sigma}_{\mathcal{T}_{e x}}, \tilde{\sigma}_{\mathcal{T}_{t o}}\right) \neq 0$, and by Lemma 3.4 $D \mathcal{F}\left(\theta_{0}\right) \tilde{\omega}=0$. Therefore, the Jacobian of the shooting mapping is singular, which achieves the proof.

\section{Sensitivity Analysis without strict complementarity at touch points}

In this section, we show how to conduct a sensitivity analysis, removing the strict complementarity hypothesis for touch points. Let $M_{0}$ be an open subset of a Banach space $M$ 
(the perturbation space). Consider, for $\mu \in M_{0}$, the family of perturbed optimal control problems:

$$
\begin{aligned}
\left(\mathcal{P}^{\mu}\right) \quad \min _{(u, y) \in \mathcal{U} \times \mathcal{Y}} & \int_{0}^{T} \tilde{\ell}(u(t), y(t), \mu) \mathrm{d} t+\tilde{\phi}(y(T), \mu) \quad \text { subject to } \\
& \dot{y}=\tilde{f}(u(t), y(t), \mu), \quad \text { a.e. } t \in[0, T] \quad ; \quad y(0)=\tilde{y}_{0}(\mu), \\
& \tilde{g}(y(t), \mu) \leq 0,
\end{aligned}
$$

where $\tilde{\ell}: \mathbb{R} \times \mathbb{R}^{n} \times M_{0} \rightarrow \mathbb{R}, \tilde{\phi}: \mathbb{R}^{n} \times M_{0} \rightarrow \mathbb{R}, \tilde{f}: \mathbb{R} \times \mathbb{R}^{n} \times M_{0} \rightarrow \mathbb{R}^{n}, \tilde{g}: \mathbb{R}^{n} \times M_{0} \rightarrow \mathbb{R}$, and $\tilde{y}_{0}: M_{0} \rightarrow \mathbb{R}^{n}$ are $C^{2}$ mappings. We denote $y_{0}^{\mu}:=\tilde{y}_{0}(\mu), \ell^{\mu}(u, y):=\tilde{\ell}(u, y, \mu)$, etc., and identify $\left(\ell^{\mu}, \phi^{\mu}, f^{\mu}, g^{\mu}, y_{0}^{\mu}\right)$ with problem $\left(\mathcal{P}^{\mu}\right)$.

We say that $\left(\mathcal{P}^{\mu}\right)$ is a $q$-stable extension of $(\mathcal{P})$, if $(\mathrm{i})$ there exist $\mu_{0} \in M_{0}$ such that $\left(\mathcal{P}^{\mu_{0}}\right)=(\mathcal{P})$, (i.e. $\ell^{\mu_{0}} \equiv \ell$, etc.), (ii) the mappings $\tilde{\ell}, \tilde{\phi}, \tilde{f}, \tilde{g}$ are $C^{2 q}$, where $q$ is the order of the state constraint of problem $(\mathcal{P})$, (iii) the state constraints are of order $q$ for all $\mu \in M_{0}$, (iv) the mappings $f^{\mu}$ are Lipschitz continuous over $\mathbb{R} \times \mathbb{R}^{n}$, uniformly over $\mu \in M_{0}$.

For each $\mu \in M_{0}$, problem $\left(\mathcal{P}^{\mu}\right)$ satisfies (A0); taking if necessary a smaller neighborhood of $\mu_{0}$, we may assume that (A1) holds as well. Given $(\mu, u, v) \in M_{0} \times \mathcal{U} \times \mathcal{V}$, denote by $\left(y_{u}^{\mu}, z_{u, v}^{\mu}\right)$ the state and linearized state, solution of:

$$
\begin{aligned}
\dot{y}_{u}^{\mu} & =f^{\mu}\left(u, y_{u}^{\mu}\right) & ; & y_{u}^{\mu}(0)=y_{0}^{\mu}, \\
\dot{z}_{u, v}^{\mu} & =f_{y}^{\mu}\left(u, y_{u}^{\mu}\right) z_{u, v}^{\mu}+f_{u}^{\mu}\left(u, y_{u}^{\mu}\right) v & ; & z_{u, v}^{\mu}(0)=0,
\end{aligned}
$$

and let $J^{\mu}(u):=\int_{0}^{T} \ell^{\mu}\left(u(t), y_{u}^{\mu}(t)\right) \mathrm{d} t+\phi^{\mu}\left(y_{u}^{\mu}(T)\right)$.

In the sequel, the bar refer to Pontryagin extremal $(\bar{u}, \bar{y})$ and associated multipliers of $(\mathcal{P}) \equiv\left(\mathcal{P}^{\mu_{0}}\right)$, while the superscript $\mu$ refers to those of $\left(\mathcal{P}^{\mu}\right)$. We make in addition to (A2)-(A4) the following assumptions:

(A7) (i) If $q=2$, (56) holds; if $q>2$, the trajectory $(\bar{u}, \bar{y})$ has no boundary arc; if $q \leq 2$, then the following strenghtening of (54)-(55) holds:

$$
\exists \beta>0 \quad(-1)^{q} \frac{\mathrm{d}^{q}}{\mathrm{~d} t^{q}} \bar{\eta}_{q}(t) \geq \beta \quad \text { for all } t \in \mathcal{I}_{b} ;
$$

(ii) If $q=1,(u, y)$ has no (non essential) touch points; if $q>1$, all touch points of $(\bar{u}, \bar{y})$ are reducible, i.e. (A6) holds.

Define the set of increasing times in $(0, T)$ of cardinal $N$ as

$$
I T_{N}:=\left\{\tau \in \mathbb{R}^{N} ; 0<\tau_{1}<\cdots<\tau_{N}<T\right\}
$$

Set $\tau_{0}:=0$ and $\tau_{N+1}:=T$. Given $\mathcal{S} \subset I T_{N}$, we have a natural isomorphism between $P C_{\mathcal{S}}^{k}[0, T]$ and $C^{k}\left([0,1] ; \mathbb{R}^{N+1}\right)$, defined by:

$$
\left\{\begin{array}{l}
\hat{\varphi}_{i}(s)=\varphi\left(\tau_{i}+\left(\tau_{i+1}-\tau_{i}\right) s\right), \quad \text { for all } s \in(0,1), \quad i=0, \ldots, N . \\
\hat{\varphi}_{i}(0)=\varphi\left(\tau_{i}^{+}\right), \hat{\varphi}_{i}(1)=\varphi\left(\tau_{i+1}^{-}\right)
\end{array}\right.
$$

INRIA 
We may therefore identify the set $P C_{N}^{k}[0, T]:=\cup\left\{P C_{\mathcal{S}}^{k}[0, T] ; \mathcal{S} \in I T_{N}\right\}$ of all possible $N$ piecewise $k$ times continuously differentiable functions, with $C^{k}\left([0,1] ; \mathbb{R}^{N+1}\right) \times I T_{N}$. The corresponding notion of convergence follows: a sequence $\varphi^{n} \in P C_{\mathcal{S}^{n}}^{k}[0, T]$ converges to $\varphi \in P C_{\mathcal{S}}^{k}[0, T]$ if $\mathcal{S}^{n} \rightarrow \mathcal{S}$ in $\mathbb{R}^{N}$ and $\hat{\varphi}^{n} \rightarrow \hat{\varphi}$ in $C^{k}\left([0,1] ; \mathbb{R}^{N+1}\right)$. Similarly, a mapping defined over an open subset $W$ of a Banach space, $W \rightarrow P C_{N}^{k}, w \mapsto \varphi^{w} \in P C_{\mathcal{S}^{w}}^{k}$ is of class $C^{k}$, if the mapping $W \rightarrow C^{k}\left([0,1], \mathbb{R}^{N+1}\right) \times \mathbb{R}^{N}, w \mapsto\left(\hat{\varphi}^{w}, \mathcal{S}_{w}\right)$ is $C^{k}$. We denote by $P C_{N}^{k, r}[0, T]=P C_{N}^{k}[0, T] \cap C^{r}[0, T]$ the subset of $P C_{N}^{k}[0, T]$ of functions having continuous derivatives on $[0, T]$ until order $r \geq 0$. The next lemma is elementary and used at the end of this section.

Lemma 4.1. Let $W$ be an open subset of a Banach space, and $W \rightarrow P C_{N}^{1,0}, w \mapsto \varphi^{w} \in$ $P C_{\mathcal{S} w}^{1,0}$ a $C^{1}$ mapping. Then the mapping $w \mapsto \varphi^{w}$ is $C^{1}$ in $L^{r}(0, T)$, for all $1 \leq r<\infty$. More precisely, for $w \in W$, let $\mathcal{S}^{w}:=\left\{\tau_{1}^{w}<\ldots<\tau_{N}^{w}\right\}$ and denote by $\left(\hat{\xi}^{w}, \sigma^{w}\right)$ the derivative in $C^{1}\left([0,1] ; \mathbb{R}^{N+1}\right) \times I T_{N}$ of the mappings $w \mapsto\left(\hat{\varphi}^{w}, \tau_{i}^{w}\right)$ at point $w$. Then the derivative $\tilde{\xi}^{w}$ in $L^{r}(0, T)$ is given by:

$$
\tilde{\xi}^{w}(t)=\hat{\xi}_{i}^{w}\left(\frac{t-\tau_{i}^{w}}{\tau_{i+1}^{w}-\tau_{i}^{w}}\right)-\dot{\varphi}^{w}(t)\left(\sigma_{i}^{w}+\frac{t-\tau_{i}^{w}}{\tau_{i+1}^{w}-\tau_{i}^{w}}\left(\sigma_{i+1}^{w}-\sigma_{i}^{w}\right)\right) \text { on }\left(\tau_{i}^{w}, \tau_{i+1}^{w}\right) .
$$

By Prop. 2.2 a regular Pontryagin extremal and its multipliers belong to the product space

$$
\mathcal{X}_{\mathcal{S}}:=P C_{\mathcal{S}}^{q, 0}[0, T] \times P C_{\mathcal{S}}^{q, 1}\left([0, T] ; \mathbb{R}^{n}\right) \times P C_{\mathcal{S}}^{1}\left([0, T], \mathbb{R}^{n *}\right) \times P C_{\mathcal{S}}^{1}[0, T],
$$

with here $\mathcal{S}=\mathcal{T}$, the finite set of its junctions times assumed to be of cardinal $N$. So let us define the union $\mathcal{X}_{N}$ of all such spaces, as well as some other sets needed latter:

$$
\begin{aligned}
\mathcal{X}_{N} & :=\cup\left\{\mathcal{X}_{\mathcal{S}} ; \quad \mathcal{S} \in I T_{N}\right\}, \\
\mathcal{X}_{\mathcal{S}}^{q} & :=P C_{\mathcal{S}}^{q}[0, T] \times P C_{\mathcal{S}}^{q, 0}\left([0, T], \mathbb{R}^{n}\right) \times P C_{\mathcal{S}}^{q}\left([0, T], \mathbb{R}^{n *}\right) \times P C_{\mathcal{S}}^{q}[0, T] \\
\mathcal{X}_{\mathcal{S}}^{1} & :=P C_{\mathcal{S}}^{q}[0, T] \times P C_{\mathcal{S}}^{q, 0}\left([0, T], \mathbb{R}^{n}\right) \times P C_{\mathcal{S}}^{1}\left([0, T], \mathbb{R}^{n *}\right) \times P C_{\mathcal{S}}^{1}[0, T], \\
\mathcal{X}_{N}^{q} & :=\cup\left\{\mathcal{X}_{\mathcal{S}}^{q}, \mathcal{S} \in I T_{N}\right\} \quad ; \quad \mathcal{X}_{N}^{1}:=\cup\left\{\mathcal{X}_{\mathcal{S}}^{1}, \mathcal{S} \in I T_{N}\right\} .
\end{aligned}
$$

Denote finally by $\mathcal{T}_{t o}^{\text {nes }}:=\mathcal{T}_{\text {to }} \backslash \mathcal{T}_{\text {to }}^{\text {ess }}$ the subset of cardinal $N_{0}$ of non essential touch points of the trajectory $(\bar{u}, \bar{y})$.

The main result of this section is the next Theorem, that gives stability results for the optimal control problem $(\mathcal{P})$, without assuming strict complementarity at touch points. Therefore we cannot apply directly the Implicit Mapping Theorem as it is done in [15, 16] or in section 3

We say that a regular Pontryagin extremal $\left(u^{\mu}, y^{\mu}\right)$ of $\left(\mathcal{P}^{\mu}\right)$ have junctions points converging to those of the trajectory $(\bar{u}, \bar{y})$, if each entry, exit and touch point of $\left(u^{\mu}, y^{\mu}\right)$ converges respectively to an entry, exit and touch point of $(\bar{u}, \bar{y})$, when $\mu \rightarrow \mu_{0}$.

Theorem 4.1. Let $(\bar{u}, \bar{y})$ be a Pontryagin extremal of $(\mathcal{P})$ satisfying $(A \mathscr{Q})-(A 4)$ and $(A 7)$. Then the following statements are equivalent: 
(i) For all q-stable extension $\left(\mathcal{P}^{\mu}\right)$, there exists a neighborhood $V_{\mu}$ of $\mu_{0}$ such that, for all $\mu \in V_{\mu}$, there exists a unique Pontryagin extremal $\left(u^{\mu}, y^{\mu}\right)$ for $\left(\mathcal{P}^{\mu}\right)$ that have junctions points converging to those of the trajectory $(\bar{u}, \bar{y})$, the mapping $\mu \mapsto\left(u^{\mu}, y^{\mu}, p^{\mu}, \eta^{\mu}\right) \in \mathcal{X}_{N}$ is Lipschitz continuous over $V_{\mu}$, and the uniform second-order growth condition below holds: there exists $c, r>0$ such that

$$
J^{\mu}(u) \geq J^{\mu}\left(u^{\mu}\right)+c\left\|u-u^{\mu}\right\|_{2}^{2}, \quad \forall u \in B_{\infty}(\bar{u}, r), g^{\mu}\left(y_{u}^{\mu}\right) \leq 0 \text { on }[0, T] .
$$

(ii) The following strong second-order sufficient condition holds:

$$
\begin{gathered}
\mathcal{J}(v, z)>0, \quad \text { for all }(v, z) \in \mathcal{V} \times \mathcal{Z} \backslash\{0\} \text { satisfying [61) and } \\
g_{y}(\bar{y}(t)) z(t)=0 \quad \text { for all } t \in \mathcal{I}_{b} \cup \mathcal{T}_{\text {to }}^{\text {ess }} .
\end{gathered}
$$

Remark 4.1. Note that condition (ii) is stronger that the following second-order characterization of quadratic growth (53) (see [3]):

$$
\begin{gathered}
\mathcal{J}(v, z)>0, \quad \text { for all }(v, z) \in \mathcal{V} \times \mathcal{Z} \backslash\{0\} \text { satisfying [61), (107) and } \\
g_{y}(\bar{y}(\tau)) z(\tau) \leq 0 \quad \text { for all } \tau \in \mathcal{T}_{\text {to }} \backslash \mathcal{T}_{\text {to }}^{\text {ess }} .
\end{gathered}
$$

We need the following notations. For $\mu$ close to $\mu_{0}$, let $\mathcal{F}(\cdot, \mu)$ be the shooting mapping (49) for problem $\left(\mathcal{P}^{\mu}\right)$, with the same structure that of the trajectory $(\bar{u}, \bar{y})$, i.e. the same number of boundary arcs and touch points and the same order of their occurence w.r.t. time. Thus non essential touch points are present in the shooting mapping, and may be active or inactive for the perturbed problem. Let $\bar{N}:=n+(q+2) N_{b}+2 N_{t o}$ denote the dimension of the shooting mapping, with $N_{b}=\operatorname{Card} \mathcal{T}_{\text {en }}=\operatorname{Card} \mathcal{T}_{\text {ex }}$ and $N_{t o}=\operatorname{Card} \mathcal{T}_{t o}$. Split $\mathcal{F}$ into two components, such that $\mathcal{F}(\cdot, \mu)=\left(\Phi(\cdot, \mu)^{*}, \Psi(\cdot, \mu)^{*}\right)^{*}$ and $\Psi$ corresponds to the component $g^{\mu}\left(y\left(\mathcal{T}_{\text {to }}^{\text {nes }}\right)\right) \in \mathbb{R}^{N_{0}}$. We consider the following problem, for $\mu$ close to $\mu_{0}$ : Find

$$
\theta=\left(\pi_{0}^{\mu *}, \nu_{\mathcal{T}_{\text {en }}}^{\mu, 1: q}, \nu_{\mathcal{T}_{\text {to }}}^{\mu}, \nu_{\mathcal{T}_{\text {to }}^{\text {nes }}}^{\mu}, \mathcal{T}_{\text {en }}^{\mu}, \mathcal{T}_{\text {ex }}^{\mu}, \mathcal{T}_{\text {to }}^{\mu, \text { ess }}, \mathcal{T}_{\text {to }}^{\mu, \text { nes }}\right) \in \Theta
$$

such that:

$$
\Phi(\theta, \mu)=0 \quad ; \quad \Psi(\theta, \mu) \in \mathbb{R}_{-}^{N_{0}} \cap\left(\nu_{\mathcal{T}_{\text {to }}^{\text {nes }}}^{\mu}\right)^{\perp} \quad ; \quad \nu_{\mathcal{T}_{\text {to }}^{\text {nes }}}^{\mu} \in \mathbb{R}_{+}^{N_{0}} .
$$

Note that the complementarity condition for essential touch points will obviously holds by continuity, since we perform a local analysis.

The point $\theta_{0}$, solution of (109) for $\mu=\mu_{0}$, is said strongly regular (Robinson [20]), if there exists a neighborhood $V_{\theta} \times V_{\delta}$ in $\mathbb{R}^{N} \times \mathbb{R}^{\bar{N}}$ of $\left(\theta_{0}, 0\right)$, such that for all $\delta \in V_{\delta}$, $\delta=\left(\delta_{1}, \delta_{2}\right) \in \mathbb{R}^{\bar{N}-N_{0}} \times \mathbb{R}^{N_{0}}$, there exists a unique solution $\theta$ in $V_{\theta}$ of:

$$
\begin{aligned}
& D_{\theta} \Phi\left(\theta_{0}, \mu_{0}\right)\left(\theta-\theta_{0}\right)-\delta_{1}=0 \\
& D_{\theta} \Psi\left(\theta_{0}, \mu_{0}\right)\left(\theta-\theta_{0}\right)-\delta_{2} \in \mathbb{R}_{-}^{N_{0}} \cap \nu_{\mathcal{T}_{\text {to }}^{\text {nes }}}^{\perp} \quad ; \quad \nu_{\mathcal{T}_{\text {to }}^{\text {nes }}} \in \mathbb{R}_{+}^{N_{0},},
\end{aligned}
$$

and the mapping $\Xi: \delta \rightarrow \theta(\delta)$ is Lipschitz continuous over $V_{\delta}$. If $\theta_{0}$ is strongly regular, then by [20], there exists a neighborhood $V_{\theta}^{\prime} \times V_{\mu}$ of $\left(\theta_{0}, \mu_{0}\right)$, such that for each $\mu \in V_{\mu}$, (109) has in $V_{\theta}^{\prime}$ a unique solution $\theta^{\mu}$ and there exists $\kappa>0$ such that for all $\mu, \mu^{\prime} \in V_{\mu}$,

$$
\left|\theta^{\mu}-\theta^{\mu^{\prime}}\right| \leq \kappa\left\|\mu-\mu^{\prime}\right\|
$$

INRIA 
and in addition, the following expansion of $\theta^{\mu}$ holds:

$$
\theta^{\mu}=\theta_{0}+\Xi\left(-D_{\mu} \mathcal{F}\left(\theta_{0}, \mu_{0}\right)\left(\mu-\mu_{0}\right)\right)+o\left(\left\|\mu-\mu_{0}\right\|\right) .
$$

\subsection{Stability Analysis (Proof of Th. 4.1)}

The first step in the proof of (ii) $\Rightarrow$ (i) in Th. 4.1) is to show that (107) implies the strong regularity property. The existence of a locally unique shooting extremal $\left(u^{\mu}, y^{\mu}\right)$ for problem $\left(\mathcal{P}^{\mu}\right)$, with a set of junctions points converging to that of $(\bar{u}, \bar{y})$, follows. The second step is to check the additional conditions of Cor. 2.1 implying that $\left(u^{\mu}, y^{\mu}\right)$ is a Pontryagin extremal. We end the proof by checking that $u^{\mu}$ satisfies the quadratic growth condition (106).

Lemma 4.2. Under the assumptions of Th. 4.1. condition (ii) of Th. 4.1 implies that $\theta_{0}$ is a strongly regular solution of (109) for $\mu=\mu_{0}$.

Proof. The proof is somewhat similar to the one of Proposition 3.1 Let $\delta=\left(\delta_{1}, \delta_{2}\right) \in$ $\mathbb{R}^{\bar{N}-N_{0}} \times \mathbb{R}^{N_{0}}$ with

$$
\delta_{1}=\left(a_{T}, b_{\mathcal{T}_{\text {en }}}^{1: q}, b_{\mathcal{T}_{\text {to }}^{\text {ess }}}^{\text {, }}, c_{\mathcal{T}_{\text {en }}}, c_{\mathcal{T}_{\text {ex }}}, c_{\mathcal{T}_{\text {to }}^{\text {ess }}}, c_{\mathcal{T}_{\text {to }}^{\text {nes }}}\right) \quad ; \quad \delta_{2}=b_{\mathcal{T}_{\text {to }}^{\text {nes }}} .
$$

Let us show that there exists a unique $\omega \in \Theta$,

$$
\omega=\left(\pi_{0}, \gamma_{\mathcal{T}_{\text {en }}}^{1: q}, \gamma_{\mathcal{T}_{\text {to }}^{\text {ess }}}, \gamma_{\mathcal{T}_{\text {to }}^{\text {nes }}}, \sigma_{\mathcal{T}_{\text {en }}}, \sigma_{\mathcal{T}_{\text {ex }}}, \sigma_{\mathcal{T}_{\text {to }}^{\text {ess }}}, \sigma_{\mathcal{T}_{\text {to }}^{\text {nes }}}\right),
$$

solution of the following relation, equivalent to (110) with $\omega=\theta-\theta_{0}$ :

$$
\begin{aligned}
& D_{\theta} \Phi\left(\theta_{0}, \mu_{0}\right) \omega-\delta_{1}=0 \\
& D_{\theta} \Psi\left(\theta_{0}, \mu_{0}\right) \omega-\delta_{2} \in \mathbb{R}_{-}^{N_{0}} \cap \gamma_{\mathcal{T}_{\text {to }}^{\text {nes }}}^{\perp} \quad ; \quad \gamma_{\mathcal{T}_{\text {to }}^{\text {nes }}} \in \mathbb{R}_{+}^{N_{0}} .
\end{aligned}
$$

Using the notation of Lemma 3.4, we will show that (113) match the optimality conditions of the following linear quadratic optimal control problem:

$$
\begin{aligned}
&\left(\mathcal{P}^{\delta}\right) \min _{v \in \mathcal{V}} \frac{1}{2} \mathcal{J}_{q}\left(v, z_{v}\right)+a_{T}^{*} z_{v}(T)+\sum_{\tau \in \mathcal{T}_{t o}} c_{\tau} \nu_{\tau} \frac{g_{y}^{(1)}(y(\tau)) z_{v}(\tau)}{\left.\frac{\mathrm{d}}{\mathrm{d} t} g^{(1)}(y)\right|_{t=\tau}} \\
& \text { subject to } \quad A v=\left(0_{L_{2}\left(\mathcal{I}_{b}\right)}, b_{\mathcal{T}_{\text {en }}^{1: q}}, b_{\mathcal{T}_{\text {to }}^{\text {ess }}}\right)^{*} ; B v \leq b_{\mathcal{T}_{\text {to }}^{\text {nes }}},
\end{aligned}
$$

where the linear operators $A, B$ are defined by:

$$
A v:=\left(\begin{array}{c}
g_{y}^{(q)}(u(\cdot), y(\cdot)) z_{v}(\cdot)+\left.g_{u}^{(q)}(u(\cdot), y(\cdot)) v(\cdot)\right|_{\mathcal{I}_{b}} \\
g_{y}^{(0: q-1)}\left(y\left(\mathcal{T}_{\text {en }}\right)\right) z_{v}\left(\mathcal{T}_{\text {en }}\right) \\
g_{y}\left(y\left(\mathcal{T}_{\text {to }}^{\text {ess }}\right)\right) z_{v}\left(\mathcal{T}_{\text {to }}^{\text {ess }}\right) \\
B v:= \\
g_{y}\left(y\left(\mathcal{T}_{\text {to }}^{\text {nes }}\right)\right) z_{v}\left(\mathcal{T}_{\text {to }}^{\text {nes }}\right) .
\end{array}\right.
$$


Being equal to $\mathcal{A}$ defined in 87], the operator $(A, B)$ is onto by Lemma 3.5. By Lemma A.3, the Legendre form $\bar{Q}(v):=\mathcal{J}_{q}\left(v, z_{v}\right)$ is coercive over Ker $A$. It follows from Lemma A.4 that problem $\left(\mathcal{P}_{\delta}\right)$ has a unique solution $u_{\delta} \in \mathcal{V}$, with unique associated Lagrange multiplier $\left(\zeta_{\delta}, \lambda_{\delta, \mathcal{T}_{e n}}^{1: q}, \lambda_{\delta, \mathcal{T}_{\text {to }}^{e s s}}, \lambda_{\delta, \mathcal{T}_{\text {to }}^{\text {nes }}}\right)$ in $L^{2}\left(\mathcal{I}_{b}\right) \times \mathbb{R}^{N_{b} \times q} \times \mathbb{R}^{N_{t o}-N_{0}} \times \mathbb{R}^{N_{0}}$, and the mapping $\delta \mapsto\left(u_{\delta}, \zeta_{\delta}, \lambda_{\delta, \mathcal{T}_{\text {en }}}^{1: q}, \lambda_{\delta, \mathcal{T}_{\text {to }}^{\text {ess }}}, \lambda_{\delta, \mathcal{T}_{t o}^{\text {nes }}}\right)$ is Lipschitz continuous. The associated state $z_{\delta}$ and costate $\pi_{\delta}$ being solution of (79)- (80) as well as (92)-(95), the mapping $\delta \mapsto\left(z_{\delta}, \pi_{\delta}\right)$ is also Lipschitz continuous for the $H^{1}$ norm, therefore also for the $P C_{\mathcal{T}}^{0}[0, T]$ norm. Consequently, by (81)- 82) $\delta \mapsto v_{\delta}\left(\tau^{ \pm}\right)$is Lipschitz continuous, for any $\tau \in \mathcal{T}$. Then, as in Lemma 3.1] we can see that $\omega$ is given by $\pi_{0}=\pi_{\delta}(0), \gamma_{\mathcal{T}_{t o}^{\text {ess }}}=\lambda_{\delta, \mathcal{T}_{t o}^{\text {ess }}}, \gamma_{\mathcal{T}_{\text {to }}^{\text {nes }}}=\lambda_{\delta, \mathcal{T}_{\text {to }}^{\text {nes }}}$, and relations (96)-(99). The existence of a unique of $\omega$ follows, as well as its Lipschitz continuity w.r.t. $\delta$.

By strong regularity, for all $\mu \in V_{\mu}$, there exists a unique solution $\theta^{\mu}$ of (109):

$$
\theta^{\mu}=\left(\pi_{0}^{\mu *}, \nu_{\mathcal{T}_{\text {en }}}^{\mu, 1: q}, \nu_{\mathcal{T}_{\text {to }}^{\text {ess }}}^{\mu}, \nu_{\mathcal{T}_{\text {to }}^{\text {nes }}}^{\mu}, \mathcal{T}_{\text {en }}^{\mu}, \mathcal{T}_{\text {ex }}^{\mu}, \mathcal{T}_{\text {to }}^{\mu, e s s}, \mathcal{T}_{\text {to }}^{\mu, n e s}\right) \in V_{\theta}^{\prime} \subset \mathbb{R}^{N} .
$$

Denote the associated trajectory and multipliers by $\left(u^{\mu}, y^{\mu}, p_{q}^{\mu}, \eta_{q}^{\mu}\right) \in \mathcal{X}_{N}$. Recall that $\Psi\left(\theta^{\mu}, \mu\right)=g^{\mu}\left(y^{\mu}\left(\mathcal{T}_{t o}^{\mu, n e s}\right)\right)$ and set

$$
\mathcal{T}_{\text {to }}^{\mu}:=\mathcal{T}_{\text {to }}^{\mu, e s s} \cup\left\{\tau \in \mathcal{T}_{\text {to }}^{\mu, n e s} ; g^{\mu}\left(y^{\mu}(\tau)\right)=0\right\} .
$$

By (109), we have $g^{\mu}\left(y^{\mu}(\tau)\right)<0$ and $\nu_{\tau}^{\mu}=0$ if $\tau \notin \mathcal{T}_{t o}^{\mu}$. Hence $\left(u^{\mu}, y^{\mu}, p_{q}^{\mu}, \eta_{q}^{\mu}\right)$ is a shooting extremal for $\left(P^{\mu}\right)$, with jumps parameters $\left(\nu_{\mathcal{T}_{e n}}^{\mu, 1: q}, \nu_{\mathcal{T}_{t o}}^{\mu}\right)$ and junctions times $\left(\mathcal{T}_{\text {en }}^{\mu}, \mathcal{T}_{\text {ex }}^{\mu}, \mathcal{T}_{t o}^{\mu}\right)$.

Consider the mapping

$$
V_{\mu} \times V_{\theta} \rightarrow \mathcal{X}_{N}^{q}, \quad(\mu, \theta) \mapsto\left(u^{\mu, \theta}, y^{\mu, \theta}, p_{q}^{\mu, \theta}, \eta_{q}^{\mu, \theta}\right)
$$

where $\left(u^{\mu, \theta}, y^{\mu, \theta}, p_{q}^{\mu, \theta}, \eta_{q}^{\mu, \theta}\right)$ is solution of (22)-(23), (25), (227), (22) and (30)-(32) for $\left(\mathcal{P}^{\mu}\right)$, with initial value of the costate, jumps parameters and junctions times given by argument $\theta$. By the Cauchy-Lipschitz Theorem, this mapping is well-defined and of class $C^{q}$ on neighborhoods $V_{\mu} \times V_{\theta}$ of $\left(\mu_{0}, \theta_{0}\right)$. It follows that the mapping

$$
V_{\mu} \times V_{\theta} \rightarrow \mathcal{X}_{N}^{1}, \quad(\mu, \theta) \mapsto\left(u^{\mu, \theta}, y^{\mu, \theta}, p^{\mu, \theta}, \eta^{\mu, \theta}\right),
$$

where $\eta_{j}^{\mu, \theta}, 0 \leq j \leq q-1, p^{\mu, \theta}$ and $\eta^{\mu, \theta}$ are defined by (35)-(37) and (20), is of class $C^{1}$.

Lemma 4.3. Under assumptions and condition (ii) of Th. 4.1, there exists a neighborhood $V_{\mu}$ of $\mu_{0}$ such that the mapping $V_{\mu} \rightarrow \mathcal{X}_{N}, \mu \mapsto\left(u^{\mu}, y^{\mu}, \eta^{\mu}, p^{\mu}\right)$ is well-defined and Lipschitz continuous on $V_{\mu}$.

Proof. Since strong regularity holds by Lemma 4.2 the mapping $\mu \mapsto \theta_{\mu}$ solution of (109), is well-defined on a neighborhood of $\mu$ and Lipschitz continuous by (111). By continuity of the mappings (117) and $\mu \mapsto \theta^{\mu}$ and Rem. 2.6. Prop. 2.4 applied to $\left(\mathcal{P}^{\mu}\right)$ and $\left(u^{\mu}, y^{\mu}\right)$ shows that $u^{\mu}$ is continuous, for all $\mu$ close enough to $\mu_{0}$, and hence, $\left(u^{\mu}, y^{\mu}\right) \in P C_{\mathcal{T}^{\mu}}^{q, 0}[0, T] \times$ $P C_{\mathcal{T}^{\mu}}^{q, 1}\left([0, T], \mathbb{R}^{n}\right)$. Reducing $V^{\mu}$ if necessary, by composition of $\mu \mapsto \theta^{\mu}$ with the $C^{1}$-mapping (117), we deduce that the mapping $\mu \mapsto\left(u^{\mu}, y^{\mu}, \eta^{\mu}, p^{\mu}\right) \in \mathcal{X}_{N}$ is Lipschitz continuous on a neighborhood of $\mu$. 
Lemma 4.4. Under assumptions and condition (ii) of Th. 4.1, the shooting extremal $\left(u^{\mu}, y^{\mu}\right)$ is a Pontryagin extremal for problem $\left(\mathcal{P}^{\mu}\right)$.

Proof. By Corollary 2.1 and (A7), we need to check (38), (39), (42) and also, when $q=2$, (50). By Lemma 4.3, (39) follows from (102). If $q=2$, (50) follows from (56). By continuity of jumps at essential touch points and definition of (109), we obtain (42). It remains to prove (38). Near an entry/exit point $\tau^{\mu}$ (when $q=1$ or 2) this is a consequence of hypothesis (58) and continuity w.r.t. $\mu$ of $u\left(\tau^{\mu \pm}\right)$. Similarly, near touch points, this follows from the reducibility hypothesis. Finally outside a small neighbourhood of contact points, we obtain that $g^{\mu}\left(u^{\mu}, y^{\mu}\right)<0$ by a standard compactness argument.

The two next lemmas extend those in [3, section 4] to the setting of perturbed optimal control problems.

Lemma 4.5. Assume that assumptions and condition (ii) of Th. 4.1 holds. Let $\left(\mathcal{P}^{\mu_{n}}\right)$ be a qstable extension with its associated Pontryagin extremal $\left(u_{n}, y_{n}\right)$ and multipliers $\left(p_{n}, \eta_{n}\right)$. Set $Q^{n}\left(v_{n}\right):=\mathcal{J}^{\mu_{n}}\left(v_{n}, z_{n}\right)$, where $\mathcal{J}^{\mu_{n}}(\cdot, \cdot)$ is given by (68) for $\left(\mathcal{P}^{\mu_{n}}\right)$, and $z_{n}:=z_{u_{n}, v_{n}}^{\mu_{n}}$ defined by (101). Assume that $v_{n} \rightarrow \bar{v} \in L^{2}$, and that $g_{y}^{\mu_{n}}\left(y_{n}(t)\right) z_{n}(t) \leq 0$, for all $t \in \operatorname{supp}\left(\eta_{n}\right)$ and all $n$. Let $\bar{z}:=z_{\bar{u}, \bar{v}}^{\mu_{0}}$. Then the following holds:

$$
\begin{gathered}
g_{y}(\bar{y}(t)) \bar{z}(t) \leq 0 \quad \text { on } \operatorname{supp}(\bar{\eta}) \\
\bar{Q}(\bar{v}) \leq \liminf Q^{n}\left(v_{n}\right) \text { and } \bar{Q}(\bar{v}) \rightarrow Q^{n}\left(v_{n}\right) \text { iff } v_{n} \rightarrow \bar{v} \text { strongly. }
\end{gathered}
$$

Proof. Since by Lemma $4.3\left(u_{n}, y_{n}\right)$ converges uniformly to $(\bar{u}, \bar{y})$, and $v_{n} \rightarrow v$, we have that $\left(z_{n}\right)$ converges weakly in $H^{1}$ to $\bar{z}$, and hence uniformly. Relation (118) follows from the convergence of entry, exit and essential touch points, strict complementarity (102), convergence of $\eta_{n}$ in $P C_{N}^{1}$, and uniform convergence of $g_{y}^{\mu_{n}}\left(y_{n}(t)\right) z_{n}$. Let us now show (119). Set $Q_{n}^{0}\left(v_{n}\right):=\int_{0}^{T} v_{n}^{*} H_{u u}^{\mu_{n}}\left(u_{n}, y_{n}, p_{n}\right) v_{n} \mathrm{~d} t$. By Lemma 4.3, uniform convergence of $z_{n}$ and convergence in $\mathcal{X}_{N}$ of $H_{u y}^{\mu_{n}}\left(u_{n}, y_{n}, p_{n}\right)$ and $H_{y y}^{\mu_{n}}\left(u_{n}, y_{n}, p_{n}\right)$, it follows easily that $Q_{n}\left(v_{n}\right)-$ $Q_{n}^{0}\left(v_{n}\right) \rightarrow \bar{Q}(\bar{v})-\bar{Q}^{0}(\bar{v})$. Writing $Q_{n}^{0}\left(v_{n}\right)=\bar{Q}^{0}\left(v_{n}\right)+r_{n}$ with $r_{n}=\int_{0}^{T} v_{n}^{*}\left(H_{u u}^{\mu_{n}}\left(u_{n}, y_{n}, p_{n}\right)-\right.$ $\left.H_{u u}(\bar{u}, \bar{y}, \bar{p})\right) v_{n} \mathrm{~d} t$, by continuity of $H_{u u}^{\mu_{n}}$ at junctions times (Lemma A.1), Lemma 4.3 implies $H_{u u}^{\mu_{n}}\left(u_{n}, y_{n}, p_{n}\right) \rightarrow H_{u u}(\bar{u}, \bar{y}, \bar{p})$ uniformly, and hence, $r_{n} \rightarrow 0$. Since by (A2), $\bar{Q}^{0}: v \mapsto$ $\int_{0}^{T} v^{*} H_{u u}(\bar{u}, \bar{y}, \bar{p}) v$ is a Legendre form, (119) follows.

We recall the reduction approach of [3, section 5.2]. When $q \geq 2$, all touch points of the trajectory $(\bar{u}, \bar{y})$ being reducible, let $\varepsilon, \delta>0$ and $V_{\mu}$ be small enough, so that, for all $\|u-\bar{u}\|_{\infty} \leq \delta$, all $\mu \in V_{\mu}$ and all $\tau_{t o} \in \mathcal{T}_{\text {to }}$, the function $g^{\mu}\left(y_{u}^{\mu}\right)$ attains its maximum over $\left[\tau_{t o}-\varepsilon, \tau_{t o}+\varepsilon\right]$ at a unique point $\tau_{u}^{\mu} \in\left(\tau_{t o}-\varepsilon, \tau_{t o}+\varepsilon\right)$. Set $\bar{I}_{t o}:=\cup_{\tau_{t o} \in \mathcal{T}_{t o}}\left(\tau_{t o}-\varepsilon, \tau_{t o}+\varepsilon\right)$ and $\bar{I}_{b}:=[0, T] \backslash \bar{I}_{t o}$. When $q=1$, set $\bar{I}_{b}:=[0, T]$ and $\bar{I}_{t o}:=\emptyset$. Then the following reduced 
problem is well-defined and locally equivalent to $\left(\mathcal{P}^{\mu}\right)$ :

$$
\begin{array}{ll}
\left(\mathcal{P}_{\text {red }}^{\mu}\right) & \min _{u \in \mathcal{B}_{\infty}(\bar{u}, \delta)} J^{\mu}(u) \quad \text { subject to } \\
& \mathcal{G}^{\mu}(u):=\left(\begin{array}{c}
\left.g\left(y_{u}\right)\right|_{\bar{I}_{b}} \\
g^{\mu}\left(y_{u}^{\mu}\left(\tau_{u}^{\mu, 1}\right)\right) \\
\vdots \\
g^{\mu}\left(y_{u}^{\mu}\left(\tau_{u}^{\mu, N_{t o}}\right)\right)
\end{array}\right) \in \mathcal{K}:=C_{-}\left[\bar{I}_{b}\right] \times \mathbb{R}_{-}^{N_{t o}} .
\end{array}
$$

The Lagrangian $\mathcal{L}^{\mu}$ of the reduced problem (120) is given, for $u \in B_{\infty}(\bar{u}, \delta)$ and a multiplier $\lambda=\left(\eta_{b}, \nu\right) \in \mathcal{M}_{+}\left[\bar{I}_{b}\right] \times \mathbb{R}_{+}^{N_{t o}}$, by

$$
\mathcal{L}^{\mu}(u, \lambda)=J^{\mu}(u)+\int_{\bar{I}_{b}} g^{\mu}\left(y_{u}^{\mu}(t)\right) \mathrm{d} \eta_{b}(t)+\sum_{i=1}^{N_{t o}} \nu_{i} g^{\mu}\left(y_{u}^{\mu}\left(\tau_{u}^{\mu, i}\right)\right) .
$$

Multipliers $\eta^{\mu}$ and $\lambda^{\mu}=\left(\eta_{b}^{\mu}, \nu^{\mu}\right)$ associated with $u^{\mu}$ in respectively problem $\left(\mathcal{P}^{\mu}\right)$ and its reduced form $\left(\mathcal{P}_{\text {red }}^{\mu}\right)$, are related by:

$$
\mathrm{d} \eta^{\mu}(t)=\mathrm{d} \eta_{b}^{\mu}(t) \text { on } \bar{I}_{b} \quad ; \quad \mathrm{d} \eta^{\mu}(t)=\sum_{i=1}^{N_{t o}} \nu_{i}^{\mu} \delta_{\tau^{\mu, i}}(t) \text { on } \bar{I}_{t o} .
$$

In addition, we can show that the reduced Lagrangian is twice Fréchet differentiable at $u^{\mu}$, and its second-order derivative satisfies, for $v \in \mathcal{V}$ :

$$
D_{u u}^{2} \mathcal{L}^{\mu}\left(u^{\mu}, \lambda^{\mu}\right)(v, v)=\mathcal{J}^{\mu}\left(v, z_{u, v}^{\mu}\right),
$$

with $\mathcal{J}^{\mu}$ given by (68). In the sequel, $T_{\mathcal{K}}(x)$ and $N_{\mathcal{K}}(x)$ denotes respectively tangent and normal cones to $\mathcal{K}$ at point $x \in \mathcal{K}$ (in the sense of convex analysis).

Lemma 4.6. Under assumptions and condition (ii) of Th. 4.1, the shooting extremal $\left(u^{\mu}, y^{\mu}\right)$ associated with $\left(\mathcal{P}^{\mu}\right)$ satisfies the uniform quadratic growth condition, and hence, is a local optimal solution of $\left(\mathcal{P}^{\mu}\right)$.

Proof. If the conclusion does not hold, then there exists a $q$-stable extension $\left(\mathcal{P}^{\mu_{n}}\right), \mu_{n} \rightarrow \mu_{0}$, with associated Pontryagin extremal and multipliers $\left(u_{n}, y_{n}, \eta_{n}, p_{n}\right) \rightarrow(\bar{u}, \bar{y}, \bar{\eta}, \bar{p})$ in $\mathcal{X}_{N}$ by Lemma 4.3 (which implies in particular $u_{n} \rightarrow \bar{u}$ in $L^{\infty}$ ), and a point $\tilde{u}_{n} \in \mathcal{U}$ feasible for $\left(P^{\mu_{n}}\right), \tilde{u}_{n} \neq u_{n}, \tilde{u}_{n} \rightarrow \bar{u}$ in $L^{\infty}$, satisfying for all $n$ :

$$
J^{\mu_{n}}\left(\tilde{u}_{n}\right) \leq J^{\mu_{n}}\left(u_{n}\right)+o\left(\left\|\tilde{u}_{n}-u_{n}\right\|_{2}^{2}\right) .
$$

Since $\lambda_{n} \in N_{\mathcal{K}}\left(\mathcal{G}^{\mu_{n}}\left(u_{n}\right)\right)$, we have (for the appropriate duality products)

$$
\left\langle\lambda_{n}, \mathcal{G}^{\mu_{n}}\left(\tilde{u}_{n}\right)-\mathcal{G}^{\mu_{n}}\left(u_{n}\right)\right\rangle \leq 0,
$$

and thus $\mathcal{L}^{\mu_{n}}\left(\tilde{u}_{n}, \lambda_{n}\right)-\mathcal{L}^{\mu_{n}}\left(u_{n}, \lambda_{n}\right) \leq o\left(\left\|\tilde{u}_{n}-u_{n}\right\|_{2}^{2}\right)$. Let $0<\varepsilon_{n}:=\left\|\tilde{u}_{n}-u_{n}\right\|_{2} \rightarrow 0$ and $v_{n}:=\varepsilon_{n}^{-1}\left(\tilde{u}_{n}-u_{n}\right)$. Since $\left\|v_{n}\right\|_{2}=1$ for all $n$, taking a subsequence if necessary, we may 
assume that $v_{n} \rightarrow \bar{v} \in \mathcal{V}$. Since $D \mathcal{G}^{\mu_{n}}\left(u_{n}\right) v_{n} \in T_{\mathcal{K}}\left(\mathcal{G}^{\mu_{n}}\left(u_{n}\right)\right)$, implying $g_{y}^{\mu_{n}}\left(y_{n}\right) z_{n} \leq 0$ on $\operatorname{supp}\left(\eta_{n}\right)$, by Lemma 4.5 we have that (118)-(119) hold. With the notations of Lemma 4.5. combining $D_{u} \mathcal{L}^{\mu_{n}}\left(u_{n}, \lambda_{n}\right)=0$ and (123) with (124)-(125), we get:

$$
Q^{n}\left(v_{n}\right)=D_{u u} \mathcal{L}^{\mu_{n}}\left(u_{n}, \lambda_{n}\right)\left(v_{n}, v_{n}\right) \leq o(1),
$$

and thus $\bar{Q}(\bar{v}) \leq 0$. Also, by (124), $D J^{\mu_{n}}\left(u_{n}\right) v_{n} \leq o(1)$, and hence,

$$
\left\langle\eta_{n}, g_{y}^{\mu_{n}}\left(y_{n}\right) z_{n}\right\rangle=\left\langle\lambda_{n}, D \mathcal{G}^{\mu_{n}}\left(u_{n}\right) v_{n}\right\rangle \geq o(1) .
$$

Passing to the limit, we obtain $\left\langle\bar{\eta}, g_{y}(\bar{y}) \bar{z}\right\rangle \geq 0$. By (118) and $\mathrm{d} \bar{\eta} \geq 0$, we deduce that $g_{y}(\bar{y}) \bar{z} \in$ $\operatorname{supp}(\bar{\eta})$, thus $\bar{v}$ and its associated linearized state $\bar{z}$ satisfy (61) and (107). Therefore condition (ii) and $\bar{Q}(\bar{v}) \leq 0$ imply $\bar{v}=0$. Since by (126), $\overline{\lim } Q^{n}\left(v_{n}\right) \leq 0$, with Lemma 4.5 . we see that $Q^{n}\left(v_{n}\right) \rightarrow 0=\bar{Q}(\bar{v})$, and hence, $v_{n} \rightarrow \bar{v}=0$, contradicting $\left\|v_{n}\right\|_{2}=1$ for all $n$.

Proof of Theorem 4.1. $(i i) \Rightarrow(i)$ is a consequence of Lemmas 4.2 to 4.6 Let us show $(i) \Rightarrow($ ii $)$. Let $\rho$ be a $C^{\infty}$ function over $\mathbb{R}, \operatorname{such}$ that $\operatorname{supp}(\rho) \subset[-1,1], \rho$ is positive over $(-1,1)$ and $\rho(1)=1$. For $\mu>0$, the function $\psi$ defined by $\psi_{\mu}(s):=\sum_{\tau \in \mathcal{T}_{t o}^{n e s}} \mu^{2 q+1} \rho\left(\frac{s-\tau}{\mu}\right)$ is $C^{2 q}$ and converge uniformly to zero, and so do its first $2 q$ derivatives. Consider the perturbed constraint mapping $g^{\mu}(y):=g(y)-\psi_{\mu}(g(y))$, and keep $\left(f, \ell, \phi, y_{0}\right)$ unchanged. Observe that $g^{\mu}$ is of order $q, g^{\mu}=g$ outside $\cup_{\tau \in \mathcal{T}_{\text {to }}^{\text {nes }}}(\tau-\mu, \tau+\mu)$ and $g^{\mu}(\bar{y})<0$ on $(\tau-\mu, \tau+\mu)$, for all $\tau \in \mathcal{T}_{t o}^{\text {nes }}$. Since touch points are isolated, for $\mu>0$ small enough, we have $g^{\mu}=g$ on $\mathcal{I}_{b} \cup \mathcal{T}_{\text {to }}^{\text {ess }}=\operatorname{supp}(\bar{\eta})$, and it is easily seen that $(\bar{u}, \bar{y})$ is a Pontryagin extremal for $\left(\mathcal{P}^{\mu}\right)$, with the same Lagrange multiplier $\bar{\eta}$ and costate $\bar{p}$. By (i), for $\mu>0$ small enough, the uniform quadratic growth (106) holds for $\left(P^{\mu}\right)$, and since assumptions (A2)-(A6) are satisfied for $\left(\mathcal{P}^{\mu}\right)$, it follows from Th. 3.3(ii) that the sufficient condition (ii) holds, which achieves the proof.

\subsection{Sensitivity Analysis}

If strong regularity holds, by (110) the mapping $\Xi: V^{\delta} \rightarrow V^{\mu} ; \delta \mapsto \theta(\delta)$ solution of (110) is positively homogeneous of degree one. It follows then from (112) that $\mu \mapsto \theta^{\mu}$ is Fréchet directionally differentiable. The directional derivatives in direction $d$ are obtained by substituting into (110) $\delta$ by $-D_{\mu} \mathcal{F}\left(\theta_{0}, \mu_{0}\right) d$. Therefore,

$$
\theta^{\mu_{0}+d}=\theta_{0}+\omega_{d}+o(\|d\|)
$$

where $\omega_{d}=\left(\pi_{d, 0}^{*}, \gamma_{d, \mathcal{T}_{e n}}^{1: q}, \gamma_{d, \mathcal{T}_{t o}}, \sigma_{d, \mathcal{T}_{e n}}, \sigma_{d, \mathcal{T}_{e x}}, \sigma_{d, \mathcal{T}_{t o}}\right)$ is as follows. Denote by $\left(v_{d}, z_{d}\right)$ and $\left(\zeta_{d}, \pi_{d}, \lambda_{d, \mathcal{T}_{e n}}^{1: q}, \lambda_{d, \mathcal{T}_{t o}}\right)$ the (unique) optimal solution and multipliers of the quadratic problem 
below:

$$
\begin{aligned}
& \left(\mathcal{P}_{d}\right) \min _{(v, z) \in \mathcal{V} \times \mathcal{Z}} \frac{1}{2} \int_{0}^{T} D_{(u, y, \mu),(u, y, \mu)}^{2} \tilde{H}\left(\bar{u}, \bar{y}, \bar{\eta}_{q}, \bar{p}_{q}, \mu_{0}\right)((v, z, d),(v, z, d)) \mathrm{d} t \\
& +\frac{1}{2} D^{2} \tilde{\phi}\left(\bar{y}(T), \mu_{0}\right)((z(T), d),(z(T), d)) \\
& +\frac{1}{2} \sum_{\tau \in \mathcal{T}_{\text {en }}} \sum_{j=1}^{q} \nu_{\tau}^{j} D^{2} \tilde{g}^{(j-1)}\left(\bar{y}(\tau), \mu_{0}\right)((z(\tau), d),(z(\tau), d)) \\
& +\frac{1}{2} \sum_{\tau \in \mathcal{T}_{t o}} \nu_{\tau}\left(D^{2} \tilde{g}\left(\bar{y}(\tau), \mu_{0}\right)((z(\tau), d),(z(\tau), d)) \frac{\left(D \tilde{g}^{(1)}\left(y(\tau), \mu_{0}\right)(z(\tau), d)\right)^{2}}{\left.\frac{\mathrm{d}}{\mathrm{d} t} g^{(1)}(y(t))\right|_{t=\tau}}\right) \\
& \text { subject to: } \quad \begin{cases}\dot{z}(t)=D \tilde{f}\left(\bar{u}, \bar{y}, \mu_{0}\right)(v, z, d) & \text { on }[0, T], \quad z(0)=0 \\
D \tilde{g}^{(0: q-1)}\left(\bar{y}(\tau), \mu_{0}\right)(z(\tau), d)=0, & \tau \in \mathcal{T}_{\text {en }}, \\
D \tilde{g}\left(\bar{y}(\tau), \mu_{0}\right)(z(\tau), d)=0, & \tau \in \mathcal{T}_{\text {to }}^{\text {ess }} \\
D \tilde{g}\left(\bar{y}(\tau), \mu_{0}\right)(z(\tau), d) \leq 0, & \tau \in \mathcal{T}_{\text {to }}^{\text {nes }} \\
D \tilde{g}(q)\left(\bar{u}, \bar{y}, \mu_{0}\right)(v, z, d)=0 & \text { on } \mathcal{I}_{b} .\end{cases}
\end{aligned}
$$

Then $\omega_{d}$ is given by: $\pi_{d, 0}=\pi_{q}(0), \gamma_{d, \mathcal{T}_{t o}}=\lambda_{d, \mathcal{T}_{t o}}$,

$$
\begin{aligned}
\sigma_{d, \tau} & =-\frac{D \tilde{g}^{(1)}\left(\bar{y}(\tau), \mu_{0}\right)\left(z_{d}(\tau), d\right)}{\left.\frac{\mathrm{d}}{\mathrm{d} t} g^{(1)}(y)\right|_{t=\tau}}, \tau \in \mathcal{T}_{t o}, \\
\sigma_{d, \tau} & =-\frac{D \tilde{g}^{(q)}\left(u(\tau), y(\tau), \mu_{0}\right)\left(v_{d}\left(\tau^{+}\right), z_{d}(\tau), d\right)}{\left.\frac{\mathrm{d}}{\mathrm{d} t} g^{(q)}(u, y)\right|_{t=\tau^{+}}}, \tau \in \mathcal{T}_{e x}, \\
\sigma_{d, \tau} & =-\frac{D \tilde{g}^{(q)}\left(\bar{u}(\tau), \bar{y}(\tau), \mu_{0}\right)\left(v_{d}\left(\tau^{-}\right), z_{d}(\tau), d\right)}{\left.\frac{\mathrm{d}}{\mathrm{d} t} g^{(q)}(u, y)\right|_{t=\tau^{-}}}, \tau \in \mathcal{T}_{\text {en }}, \\
\gamma_{d, \tau}^{1} & =\lambda_{d, \tau}^{1}, \quad \gamma_{d, \tau}^{j}=\lambda_{d, \tau}^{j}-\nu_{\tau}^{j-1} \sigma_{d, \tau}, \quad j=2, \ldots, q, \tau \in \mathcal{T}_{\text {en }} .
\end{aligned}
$$

Once we have the expressions for the directional derivatives of the shooting paramaters, by composition with the Fréchet derivatives of the $C^{1}$-mapping (117) at point $\left(\omega_{d}, d\right)$, we obtain the expressions of the directional derivatives, in $\mathcal{X}_{N}$, of the mapping $\mu \mapsto\left(u^{\mu}, y^{\mu}, \eta^{\mu}, p^{\mu}\right)$. By Lemma 4.1, we obtain then easily the expression of the directional derivatives of the control and state in $L^{r}(0, T) \times W^{1, r}\left(0, T ; \mathbb{R}^{n}\right)$, for all $1 \leq r<\infty$.

Corollary 4.1. If either point (i) or (ii) of Theorem 4.1 is satisfied, then there exists a neighborhood $V_{\mu}$ of $\mu$, such that the mapping $V_{\mu} \rightarrow \mathcal{X}_{N}, \mu \mapsto\left(u^{\mu}, y^{\mu}, \eta^{\mu}, p^{\mu}\right)$ is Fréchetdirectionaly differentiable on $V_{\mu}$. In addition, the directional derivative of the mapping $\mu \rightarrow\left(u^{\mu}, y^{\mu}\right)$ in $L^{r}(0, T) \times W^{1, r}\left(0, T ; \mathbb{R}^{n}\right), 1 \leq r<\infty$, at point $\mu_{0}$ in direction $d$, is the optimal solution $\left(v_{d}, z_{d}\right)$ of problem $\left(\mathcal{P}_{d}\right)$.

We end the paper by a remark related to the ill-posedness of the shooting algorithm for state constraint of order $q \geq 3$, when boundary arcs are present (see Th. 3.2).

INRIA 
Remark 4.2. Existence of regular boundary arcs for constraints of order $q \geq 3$. Contrary to some conjectures in the literature, regular boundary arcs can occur for state constraint of all order. Take for example the problem:

$$
\begin{array}{cl}
\left(P_{q}\right) & \min _{(u, y) \in L^{\infty}(0, T) \times W^{q, \infty}(0, T)} \int_{0}^{T}\left(y(t)+\frac{u^{2}(t)}{2}\right) \mathrm{d} t \\
\text { subject to } & y^{(q)}(t)=u(t) ; y(0)=y_{1}^{0} ; \dot{y}(0)=y_{2}^{0} ; \ldots ; y^{(q-1)}(0)=y_{q}^{0} ; \\
& y(t) \geq 0, \quad t \in[0, T] .
\end{array}
$$

It is easy to check that, for $\tau \in(0, T), y$ defined by $y(t)=0$ on $[\tau, T]$ and

$$
y(t)=\left\{\begin{array}{ll}
\frac{(t-\tau)^{2 q}}{(2 q) !} & \text { if } q \text { is odd } \\
-\frac{(t-\tau)^{2 q}}{(2 q) !}-\nu \frac{(t-\tau)^{2 q-1}}{(2 q-1) !} & \text { if } q \text { is even }
\end{array} \quad \text { on }[0, \tau],\right.
$$

is, for $\nu>\tau / 2 q$ if $q$ is even and appropriate initial conditions when $q \geq 3$, a solution that satisfies all necessary optimality conditions and hence, by convexity of the problem, an optimal solution with a regular entry point $\tau$, and strict complementarity holds since $\eta_{0}(t)=1$ on $(\tau, T]$.

Robbins in [19] studies this example for generic initial conditions and shows that the optimal trajectory has a boundary arc, but the latter is not regular. Its entry point is the limit of an infinite number of touch points, with a geometric decreasing of the length of the interior arcs. Regular boundary arcs corresponds to the case when the multiplier of the geometric sequence is equal to zero, for a specific subset of initial conditions. Therefore, we see on that example, though satisfying all regularity assumptions (A0)-(A3), that the structure of boundary arcs is not stable under perturbations of the initial condition when $q \geq 3$, which illustrates why the shooting algorithm should be ill-posed in that case.

\section{A Technical lemmas}

The two next lemmas follows immediatly from the junctions conditions established in [13, 17].

Lemma A.1. Let $(u, y)$ be a regular Pontryagin extremal satisfying (A2)-(A4). Then the function $t \mapsto H_{u u}(u(t), y(t), p(t)$ is continuous on $[0, T]$.

Proof. Let $\tau \in \mathcal{T}$. Since $u$ is continuous by Prop. 2.2, we have:

$$
\left[H_{u u}(u(\tau), y(\tau), p(\tau))\right]=[p(\tau)] f_{u u}(u(\tau), y(\tau))=-\nu_{\tau} g_{u u}^{(1)}(u(\tau), y(\tau))=0,
$$

since either $\nu_{\tau}=0$ when $q=1$ or $g_{u}^{(1)} \equiv 0$ when $q>1$.

Lemma A.2. Let $(u, y)$ be a regular Pontryagin extremal, satisfying (A2)-(A4), and $\tau \in$ $\mathcal{T}_{\text {en }} \cup \mathcal{T}_{\text {ex }}$ an entry/exit time. The following conditions are equivalent:

(i) (58) holds at $\tau$; (ii) if $q$ is odd, $\lim _{t \rightarrow \tau ; t \in \mathcal{I}_{b}} \eta_{0}(t)>0$; if $q$ is even, $\nu_{\tau}>0$. 
Proof. Define the mappings $\left(A_{l}\right)_{0 \leq l \leq q}:[0, T] \backslash \mathcal{T} \mapsto \mathbb{R}^{n}$ by (33) and $\left(a_{l}\right)_{0 \leq l \leq q}:[0, T] \backslash \mathcal{T} \mapsto \mathbb{R}$ by:

$$
a_{0}(t)=\ell_{u}(u(t), y(t)) \quad ; \quad a_{l}(t)=\ell_{y}(u(t), y(t)) A_{l-1}(t)-\dot{a}_{l-1}(t) \quad l=1, \ldots, q .
$$

Then it can be seen by (34) (see [17]) that for all $t \in[0, T] \backslash \mathcal{T}$, we have

$$
\begin{aligned}
& 0=\frac{\mathrm{d}^{j}}{\mathrm{~d} t^{j}} H_{u}(u(t), y(t), p(t))=(-1)^{j}\left(a_{j}(t)+p(t) A_{j}(t)\right) ; j=0, \ldots, q-1, \\
& 0=\frac{\mathrm{d}^{q}}{\mathrm{~d} t^{q}} H_{u}(u(t), y(t), p(t))=(-1)^{q}\left(a_{q}(t)+p(t) A_{q}(t)+\frac{\mathrm{d} \eta}{\mathrm{d} t} g_{u}^{(q)}(u(t), y(t))\right) .
\end{aligned}
$$

Since the derivatives of the control are continuous until order $q-2$, the functions $a_{j}$ and $A_{j}$ are continuous for $j=0, \ldots, q-2$, and it is then easily seen, since $u$ is continuous, that the jump of $A_{q-1}$ and $a_{q-1}$ at $\tau \in \mathcal{T}$, when $q$ is even, are given respectively by

$$
\left[A_{q-1}(\tau)\right]=(-1)^{q-1} f_{u u}(u(\tau), y(\tau)) ; \quad\left[a_{q-1}(\tau)\right]=(-1)^{q-1} \ell_{u u}(u(\tau), y(\tau))\left[u^{(q-1)}(\tau)\right] .
$$

Taking the jump in (132) at $\tau$ for $j=q-1$ yields then:

$$
0=(-1)^{q-1} H_{u u}\left(u(\tau), y(\tau), p\left(\tau^{+}\right)\right)\left[u^{(q-1)}(\tau)\right]-\nu_{\tau} g_{y}(y(\tau)) A_{q-1}\left(\tau^{-}\right) .
$$

By (34), we have $g_{y}(y(\tau)) A_{q-1}\left(\tau^{ \pm}\right)=g_{u}^{(q)}(u(\tau), y(\tau))$, so we obtain, when $q$ is even:

$$
\nu_{\tau}=(-1)^{q-1} \frac{H_{u u}\left(u(\tau), y(\tau), p\left(\tau^{+}\right)\right)\left[u^{(q-1)}(\tau)\right]}{g_{u}^{(q)}(u(\tau), y(\tau))} .
$$

It follows that $\nu_{\tau}>0$ iff $u^{(q-1)}$ is discontinuous at $\tau$, which is equivalent to say that (558) holds (when $q$ is even). When $q$ is odd, $u^{(q-1)}, a_{q-1}$ and $A_{q-1}$ are continuous (and $\nu_{\tau}=0$ ). Taking the jump in (133), we obtain:

$$
0=(-1)^{q} H_{u u}(u(\tau), y(\tau), p(\tau))\left[u^{(q)}(\tau)\right]+\left[\eta_{0}(\tau)\right] g_{u}^{(q)}(u(\tau), y(\tau)) .
$$

As a consequence, we have $\eta_{0}\left(\tau^{ \pm}\right)>0$ at an entry/exit point, where $\tau^{ \pm}$stands for $\tau^{+}$if $\tau \in \mathcal{T}_{\text {en }}$ and $\tau^{-}$if $\tau \in \mathcal{T}_{\text {ex }}$ iff $u^{(q)}$ is discontinuous at $\tau$, and hence iff (58) holds.

The next two lemmas recall classical results.

Lemma A.3. Let $X$ be a Hilbert space and $Q$ a Legendre form over $X$. Let $A$ be a continuous linear operator over $X$. The following assertions are equivalent:

(i) $Q(v)>0$, for all $v \in \operatorname{Ker} A$;

(ii) There exists $\alpha>0$ such that $Q(v) \geq \alpha\|v\|_{2}^{2}$, for all $v \in \operatorname{Ker} A$.

Lemma A.4. Let $X$ be a Hilbert space and $Y$ a Banach space, $H: X \rightarrow X^{*} \equiv X$ a selfadjoint continuous linear operator, and $A: X \rightarrow Y$ and $B: X \rightarrow \mathbb{R}^{r}, r \in \mathbb{N}$, continuous linear operators. Assume that:

(i) $\quad \exists \alpha>0 \quad\langle H x, x\rangle \geq \alpha\|x\|^{2}, \quad$ for all $x \in \operatorname{Ker} A$,

(ii) The operator $(A, B): X \rightarrow Y \times \mathbb{R}^{r}$ is onto. 
Then, for all $\left(x^{*}, y, \delta\right) \in X^{*} \times Y \times \mathbb{R}^{r}$, there exists a unique $\left(x, y^{*}, \nu\right) \in X \times Y^{*} \times \mathbb{R}^{r *}$, solution of:

$$
\left\{\begin{array}{l}
H x+A^{*} y^{*}+B^{*} \nu=x^{*} \\
A x=y \\
B x \leq \delta, \quad \nu \geq 0, \quad \nu(B x-\delta)=0
\end{array}\right.
$$

and the mapping $\left(x^{*}, y, \delta\right) \mapsto\left(x, y^{*}, \nu\right)$, where $\left(x, y^{*}, \nu\right)$ is solution of 135), is Lipschitz continuous.

\section{References}

[1] D. Augustin and H. Maurer. Computational sensitivity analysis for state constrained optimal control problems. Ann. Oper. Res., 101:75-99, 2001.

[2] P. Berkmann and H.J. Pesch. Abort landing in windshear: optimal control problem with third-order state constraint and varied switching structure. J. of Optimization Theory and Applications, 85, 1995.

[3] F. Bonnans and A. Hermant. No-gap second-order optimality conditions for optimal control problems with a single state constraint and control. Research Report 5837, INRIA, Février 2006.

[4] J.F. Bonnans and A. Shapiro. Perturbation analysis of optimization problems. SpringerVerlag, New York, 2000.

[5] A. E. Bryson and Y.-C. Ho. Applied optimal control. Hemisphere Publishing, New-York, 1975.

[6] A.E. Bryson, W.F. Denham, and S.E. Dreyfus. Optimal programming problems with inequality constraints I: necessary conditions for extremal solutions. AIAA Journal, 1:2544-2550, 1963.

[7] R. Bulirsch, F. Montrone, and H. J. Pesch. Abort landing in the presence of windshear as a minimax optimal control problem. I. Necessary conditions. J. of Optimization Theory and Applications, 70:1-23, 1991.

[8] F.H. Clarke. Optimization and nonsmooth analysis. Wiley, New York, 1983.

[9] A.L. Dontchev and W.W. Hager. Lipschitzian stability for state constrained nonlinear optimal control. SIAM J. on Control and Optimization, 36(2):698-718 (electronic), 1998.

[10] Halina Frankowska and Barbara Kas̀kosz. A maximum principle for differential inclusion problems with state constraints. Systems Control Lett., 11:189-194, 1988.

[11] R.F. Hartl, S.P. Sethi, and R.G. Vickson. A survey of the maximum principles for optimal control problems with state constraints. SIAM Review, 37:181-218, 1995.

$\mathrm{RR} \mathrm{n}^{\circ} 5889$ 
[12] A.D. Ioffe and V.M. Tihomirov. Theory of Extremal Problems. North-Holland Publishing Company, Amsterdam, 1979. Russian Edition: Nauka, Moscow, 1974.

[13] D.H. Jacobson, M.M. Lele, and J.L. Speyer. New necessary conditions of optimality for control problems with state-variable inequality contraints. J. of Mathematical Analysis and Applications, 35:255-284, 1971.

[14] K. Malanowski. Sufficient optimality conditions for optimal control subject to state constraints. SIAM J. Control Optim., 35, 1997.

[15] K. Malanowski and H. Maurer. Sensitivity analysis for state constrained optimal control problems. Discrete and Continuous Dynamical Systems, 4:241-272, 1998.

[16] K. Malanowski and H. Maurer. Sensitivity analysis for optimal control problems subject to higher order state constraints. Annals of Operations Research, 101:43-73, 2001. Optimization with data perturbations, II.

[17] H. Maurer. On the minimum principle for optimal control problems with state constraints. Schriftenreihe des Rechenzentrum 41, Universität Münster, 1979.

[18] H. J. Pesch. A practical guide to the solution of real-life optimal control problems. Control and Cybernetics, 23:7-60, 1994.

[19] H.M. Robbins. Junction phenomena for optimal control with state-variable inequality constraints of third order. J. of Optimization Theory and Applications, 31:85-99, 1980.

[20] S.M. Robinson. Strongly regular generalized equations. Mathematics of Operations Research, 5:43-62, 1980.

[21] J. Stoer and R. Bulirsch. Introduction to Numerical Analysis. Springer-Verlag, NewYork, 1993. 


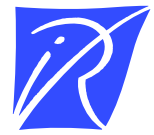

Unité de recherche INRIA Rocquencourt

Domaine de Voluceau - Rocquencourt - BP 105 - 78153 Le Chesnay Cedex (France)

Unité de recherche INRIA Futurs : Parc Club Orsay Université - ZAC des Vignes

4, rue Jacques Monod - 91893 ORSAY Cedex (France)

Unité de recherche INRIA Lorraine : LORIA, Technopôle de Nancy-Brabois - Campus scientifique

615, rue du Jardin Botanique - BP 101 - 54602 Villers-lès-Nancy Cedex (France)

Unité de recherche INRIA Rennes : IRISA, Campus universitaire de Beaulieu - 35042 Rennes Cedex (France)

Unité de recherche INRIA Rhône-Alpes : 655, avenue de l'Europe - 38334 Montbonnot Saint-Ismier (France)

Unité de recherche INRIA Sophia Antipolis : 2004, route des Lucioles - BP 93 - 06902 Sophia Antipolis Cedex (France) 